\title{
Computing undercompressive waves with the random choice scheme. Nonclassical shock waves
}

\author{
C. Chalons ${ }^{\dagger}$ \\ O.N.E.R.A., B.P. 72, 29 avenue de la Division Leclerc, 92322 Châtillon Cedex, France \\ Centre de Mathématiques Appliquées \& CNRS, U.M.R. 7641, Ecole Polytechnique, \\ 91128 Palaiseau Cedex, France \\ AND \\ P. G. LEFLOCH \\ Centre de Mathématiques Appliquées \& CNRS, U.M.R. 7641, Ecole Polytechnique, \\ 91128 Palaiseau Cedex, France
}

[Received 27 March 2002 and in revised form 8 November 2002]

\begin{abstract}
For several nonlinear hyperbolic models of interest we investigate the stability and largetime behavior of undercompressive shock waves characterized by a kinetic relation. The latter are considered as interfaces between two materials with distinct constitutive relations. We study nonclassical entropy solutions to scalar conservation laws with concave-convex flux-function and a non-genuinely nonlinear, strictly hyperbolic model of two conservation laws arising in nonlinear elastodynamics. We use Glimm's random choice scheme but we replace the classical Riemann solver with the nonclassical one described recently in [21, 24]. Our numerical experiments demonstrate the robustness and accuracy of the random choice scheme for computing nonclassical shock waves which are known to be very sensitive to dissipation and dispersion mechanisms. In this paper, we study carefully various issues related to nonclassical shocks and their stability under perturbations. This numerical study yields important hints for further theoretical investigation on, for instance, the double $N$-wave pattern put forward when studying the time-asymptotic behavior of periodic nonclassical solutions.
\end{abstract}

\section{Introduction}

We are interested in computing weak solutions of the initial-value problem for one-dimensional, nonlinear systems of conservation laws of the form

$$
\begin{gathered}
\partial_{t} u+\partial_{x} f(u)=0, \quad u(x, t) \in \mathbb{R}^{N}, x \in \mathbb{R}, t>0, \\
u(x, 0)=u_{0}(x), \quad x \in \mathbb{R} .
\end{gathered}
$$

Such systems arise in a broad spectrum of problems in compressible fluid dynamics, nonlinear elastodynamics, etc. Due to the nonlinearity $f: \mathbb{R}^{N} \rightarrow \mathbb{R}^{N}$, solutions are generally discontinuous and it is well known that weak solutions (in the integral sense) are not uniquely determined by their

\footnotetext{
†Email: chalons@onera.fr

Email: lefloch@cmap.polytechnique.fr
} 
initial data $u_{0}: \mathbb{R} \rightarrow \mathbb{R}^{N}$, and must be constrained by an entropy inequality of the form

$$
\partial_{t} U(u)+\partial_{x} F(u) \leqslant 0
$$

in the weak sense, where $(U, F)$ denotes an entropy-entropy flux pair for the system (1.1), satisfying by definition $D F^{T}=D U^{T} D f$. Here, we will focus on solutions containing undercompressive shock waves: the number of characteristics (wave modes) impinging on the discontinuity is less than what is usually required for the linearized stability. Undercompressive waves turn out to be not uniquely determined by $(1.3)$. However, under some assumptions to be specified in several examples below, the uniqueness of the entropy solution of the problem $(1.1)-(1.3)$ is ensured when a kinetic relation is added along each undercompressive discontinuity connecting a left-hand state $u_{-}$to a right-hand state $u_{+}$:

$$
u_{+}=\varphi^{b}\left(u_{-}\right) .
$$

The kinetic function $\varphi^{b}: \mathbb{R}^{N} \rightarrow \mathbb{R}^{N}$ is a Lipschitz continuous mapping satisfying the basic conditions

$$
-\lambda^{\mathrm{b}}\left(u_{-}\right)\left(\varphi^{\mathrm{b}}\left(u_{-}\right)-u_{-}\right)+f\left(\varphi^{\mathrm{b}}\left(u_{-}\right)\right)-f\left(u_{-}\right)=0
$$

and

$$
-\lambda^{b}\left(u_{-}\right)\left(U\left(\varphi^{b}\left(u_{-}\right)\right)-U\left(u_{-}\right)\right)+F\left(\varphi^{b}\left(u_{-}\right)\right)-F\left(u_{-}\right) \leqslant 0,
$$

where $\lambda^{b}\left(u_{-}\right)$denotes the speed of propagation. The nonclassical shocks are also referred to as phase transition boundaries or interfaces. For a complete discussion of the notion of kinetic relation we refer to the recent monograph [21] and the references therein.

Our general aim is to investigate numerically the stability and large-time behavior of undercompressive waves using, as a tool, Glimm's random choice scheme [9]. In the present paper, we focus attention on nonclassical shocks of strictly hyperbolic systems which fail to be globally genuinely nonlinear in the sense of Lax. That is, the Jacobian matrix $D f(u)$ admits real and distinct eigenvalues $\lambda_{j}(u)$ and independent eigenvectors $r_{j}(u)(1 \leqslant j \leqslant N)$ and for one wave family at least the product $\nabla \lambda_{j}(u) \cdot r_{j}(u)$ does not keep a constant sign. Specifically, we consider the scalar conservation law with cubic flux-function (Section 2) and a hyperbolic model arising in nonlinear elastodynamics (Section 3). In a next study, we will consider phase boundaries of a hyperbolicelliptic model arising in phase dynamics.

Let us recall some basic features of the random choice method. Glimm's scheme [9, 26, 5] is based on an equidistributed sequence $\left(a_{n}\right)_{n=1,2, \ldots}$ of values in the interval $(0,1)$ satisfying, by definition, for each $J \subset(0,1)$,

$$
\frac{1}{m} \operatorname{card}\left\{n \mid 1 \leqslant n \leqslant m \text { and } a_{n} \in J\right\} \rightarrow \operatorname{meas}(J)
$$

as $m \rightarrow \infty$. The scheme is based on solving Riemann problems corresponding to the piecewise constant initial data:

$$
u_{0}(x)= \begin{cases}u_{L}, & x<0 \\ u_{R}, & x>0\end{cases}
$$

where $u_{L}$ and $u_{R}$ are constant states. The Riemann solution has a rather simple form: explicitly, it is made of several shock waves and rarefaction waves separated by constant states. Glimm's scheme proceeds as follows. First, the initial data $u_{0}$ in (1.2) is replaced by a piecewise constant approximation $u_{0}^{\Delta x}$ where $\Delta x>0$ represents the constant mesh size of a regular mesh $x_{k}=k \Delta x$ for $k=\ldots,-1,0,1, \ldots$ At each initial discontinuity a Riemann problem is solved locally, using a classical or a nonclassical Riemann solver. (Such solvers are derived in [21].) At some sufficiently 
small time $\Delta t$ satisfying the stability condition

$$
\Delta t \sup \left|\lambda_{j}(u)\right|<\frac{\Delta x}{2},
$$

a value is picked up "randomly" within each local Riemann solutions. This provides us with the new approximation at time $\Delta t$. The construction is continued inductively in time until the approximation $u^{\Delta x, \Delta t}=u^{\Delta x, \Delta t}(x, t)$ is determined for all times. Throughout the present paper, following a proposal by Collela [5] we use the van der Corput random sequence (see Test 1 below).

All the figures in this paper represent plots $x \mapsto u(x, t)$ of $[1.1$ for various examples of equations and systems and various initial data. The time $t$ is fixed, and the horizontal and vertical axes always represent the space coordinate and conservative variable, respectively (unless otherwise stated). In our study of the time-asymptotic behavior below, we often indicate the number of iterations needed rather than the time $t$ at which the result is shown. Note that we always use a CFL number equal to 0.5 (as in (1.8) above).

Note added in proof. The double N-wave pattern put forward numerically in this paper was first discovered analytically by C. M. Dafermos in "Large time behavior of periodic solutions of hyperbolic systems of conservation laws", J. Differential Equations 121 (1995), 183-202, and in "Regularity and large time behavior of a conservation law without convexity", Proc. Roy. Soc. Edinburgh 99 (1985), 201-239.

\section{Conservation law with cubic flux}

To begin with, we consider the nonlinear conservation law

$$
\partial_{t} u+\partial_{x} u^{3}=0, \quad u(x, t) \in \mathbb{R},
$$

which is the simplest example of a nonlinear hyperbolic equation which fails to be globally genuinely nonlinear. Following [12,21] we consider solutions satisfying the conservation law (2.1) in the integral sense, the initial condition

$$
u(x, 0)=u_{0}(x), \quad x \in \mathbb{R},
$$

the entropy inequality

$$
\partial_{t} u^{2}+\frac{3}{2} \partial_{x} u^{4} \leqslant 0
$$

in the integral sense and (for definiteness in our numerical investigations) a kinetic function in the form

$$
\varphi^{b}(u)=-\beta u, \quad \beta \in[1 / 2,1) .
$$

The parameter $\beta$ is a measure of "how far" the solution is from the classical regime. Precisely, as $\beta \rightarrow 1 / 2$, the Riemann solution converges (pointwise, away from jump discontinuities) to the classical entropy solution selected by the Oleĭnik entropy inequalities. The choice $\beta=1$ is not truly allowed in the general theory of [21], but is a limiting case, referred to as the maximally dissipative kinetic relation: the corresponding shock waves have zero-entropy dissipation and achieve the largest propagation speed among all admissible nonclassical shocks.

Observe that 2.4] satisfies the standard assumption made on kinetic functions in [21]:

$$
\begin{array}{ll}
-u<\varphi^{b}(u) \leqslant-u / 2, & u>0, \\
-u / 2 \leqslant \varphi^{b}(u)<-u, & u<0 .
\end{array}
$$


We also set

$$
\varphi^{\sharp}(u)=-u-\varphi^{\mathrm{b}}(u) .
$$

Given constant states $u_{L}$ and $u_{R}$, the nonclassical Riemann solver associated with 2.1)-2.4 and the initial data

$$
u_{0}(x)= \begin{cases}u_{L}, & x<0, \\ u_{R}, & x>0,\end{cases}
$$

is given as follows, assuming for definiteness that $u_{L}>0$ :

(1) If $u_{R} \geqslant u_{L}$, the solution is a rarefaction wave connecting $u_{L}$ to $u_{R}$.

(2) If $u_{R} \in\left[\varphi^{\sharp}\left(u_{L}\right), u_{L}\right)$, the solution is a classical shock wave connecting $u_{L}$ to $u_{R}$.

(3) If $u_{R} \in\left(\varphi^{\mathrm{b}}\left(u_{L}\right), \varphi^{\sharp}\left(u_{L}\right)\right)$, the solution contains a nonclassical shock connecting $u_{L}$ to $\varphi^{\mathrm{b}}\left(u_{L}\right)$, followed by a classical shock connecting $\varphi^{\mathrm{b}}\left(u_{L}\right)$ to $u_{R}$.

(4) If $u_{R} \leqslant \varphi^{b}\left(u_{L}\right)$, the solution contains a nonclassical shock connecting $u_{L}$ to $\varphi^{\mathrm{b}}\left(u_{L}\right)$, followed by a rarefaction connecting $\varphi^{\mathrm{b}}\left(u_{L}\right)$ to $u_{R}$.

\section{Test 1: Van der Corput sequence}

We start by comparing the efficiency of the van der Corput sequence versus an "arbitrary" random sequence. Recall that the van der Corput sequence is defined by

$$
a_{n}=\sum_{k=0}^{m} i_{k} 2^{-(k+1)},
$$

where $n=\sum_{k=0}^{m} i_{k} 2^{k}, i_{k}=0,1$, denotes the binary expansion of the integers $n=1,2, \ldots$ The first few elements of this sequence are

$$
\begin{aligned}
& 1=1_{2}, \quad a_{1}=0.5, \quad 2=10_{2}, \quad a_{2}=0.25, \\
& 3=11_{2}, \quad a_{3}=0.75, \quad 4=100_{2}, \quad a_{4}=0.125 \text {, } \\
& 5=101_{2}, \quad a_{5}=0.625, \quad 6=110_{2}, \quad a_{6}=0.375 \text {, } \\
& 7=111_{2}, \quad a_{7}=0.875, \quad 8=1000_{2}, \quad a_{8}=0.0625 .
\end{aligned}
$$

This sequence is equidistributed within the interval. In particular one can prove that

$$
\begin{array}{ll}
a_{i}<0.5, & i \text { even, } \\
a_{i}>0.5, & i \text { odd. }
\end{array}
$$

Excellent numerical results in the smooth parts of the solution are obtained with this sequence, as we now illustrate.

Consider the Riemann data

$$
u_{0}(x)= \begin{cases}2, & x<0 \\ 4, & x>0\end{cases}
$$

The solution is made of a single rarefaction wave and the kinetic function is here irrelevant. The mesh contains 300 points and the solution is plotted in Figure 1 at time $t=0.007$. We observe that the van der Corput sequence gives much more accurate and regular results than an arbitrary random sequence. That is the reason why from now on we will always make use of this sequence in the next experiments. 


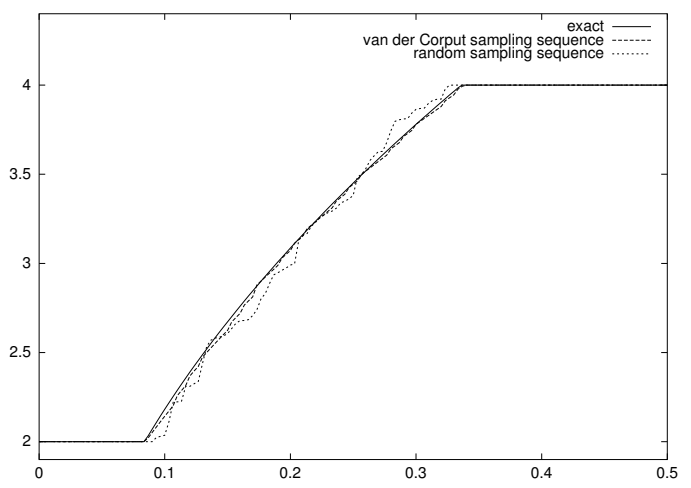

FIG. 1. Two random sequences.

Test 2: The Riemann solver

Next, we display the four qualitative behaviors of the Riemann solution for the problem (2.1)-2.4, with

$$
u_{0}(x)= \begin{cases}u_{L}, & x<-0.375 \\ u_{R}, & x>-0.375\end{cases}
$$

and the parameter value $\beta=2 / 3$. The mesh contains 300 points and the numerical solutions are plotted in Figures 2.

- Figure 2A: A single classical shock wave. We plot here the numerical solution at time $t=0.05$, corresponding to the data $u_{L}=2$ and $u_{R}=1$.

- Figure 2B: A single rarefaction wave. We plot the solution obtained with $u_{L}=1$ and $u_{R}=2$, at time $t=0.05$.

- Figure 2C: A nonclassical shock wave followed by a classical shock wave. We plot the numerical solution at time $t=0.6$, corresponding to the Riemann data $u_{L}=1$ and $u_{R}=-0.4$.
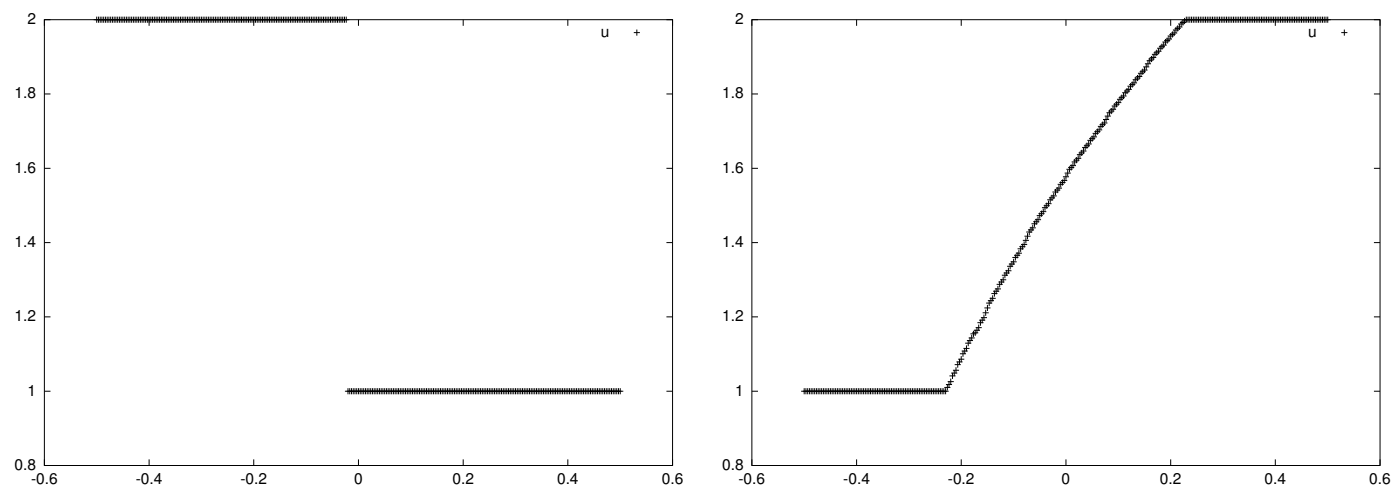

FIG. 2A \& 2B. Typical solutions: single classical wave. 

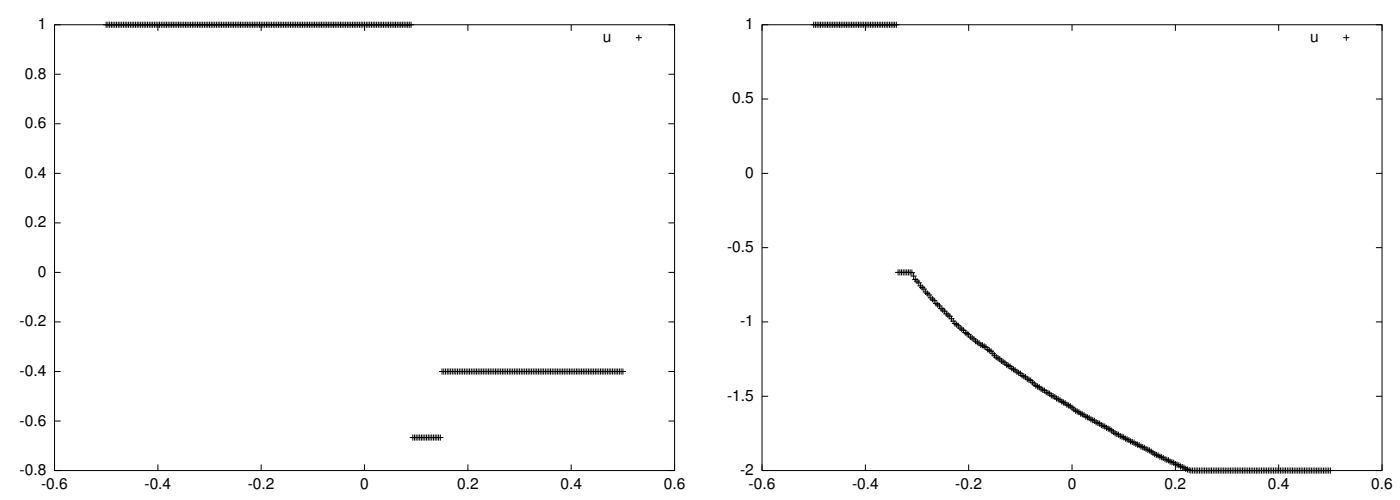

FIG. 2C \& 2D. Typical solutions: classical wave and nonclassical wave.

- Figure 2D: A nonclassical shock wave followed by a rarefaction wave. We plot the numerical solution at time $t=0.05$, corresponding to $u_{L}=1$ and $u_{R}=-2$.

We observe that the numerical solutions agree with the above nonclassical Riemann solver. Shock waves are represented by sharp discontinuities which is a main feature of the random choice scheme: all values taken by the numerical solutions are actual values taken by the exact solutions. The location of the discontinuities however depends on the random sequence. By contrast, finite difference schemes generate intermediate points within a propagating discontinuity and a discrete numerical shock profile (see for instance [4] and the references therein).

\section{Test 3: Splitting of shock waves}

We present three related approaches illustrating an important numerical difficulty encountered with nonclassical shock waves: the nonclassical Riemann solver depends continuously upon its end states in the $L^{1}$ norm but not in the pointwise sense. As a matter of fact, this is a major difficulty dealt with in the general existence theory developed in [21]. Because of this lack of continuity, some "spikes" may be observed in the numerical solution, which are genuine features of the exact solution [21]. Interestingly enough, as demonstrated by our experiments, Glimm's scheme allows us to determine the exact value of the solution "inside" the spikes. In general, this feature of the exact solution is more difficult to observe properly using finite difference schemes which smooth out discontinuities and spikes; see [4], [13], [23]).

The Riemann problem associated with

$$
u_{0}(x)= \begin{cases}u_{L}, & x<-0.375, \\ \varphi^{\sharp}\left(u_{L}\right), & x>-0.375,\end{cases}
$$

can be solved with a single classical shock wave.

- Figure 3A: Small perturbations of a classical shock. If we perturb the above initial data by an arbitrarily small amount $\epsilon>0$, specifically

$$
u_{0}^{\epsilon}(x)= \begin{cases}u_{L}, & x<-0.375, \\ \varphi^{\sharp}\left(u_{L}\right)-\epsilon, & x>-0.375,\end{cases}
$$




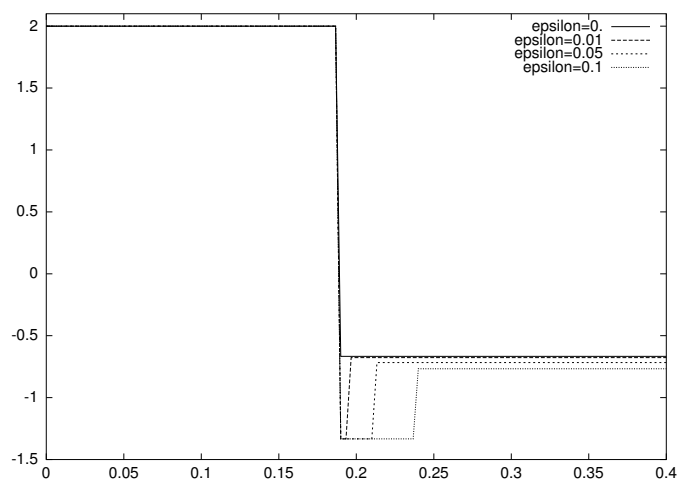

FIG. 3A. Small perturbations of a classical shock.
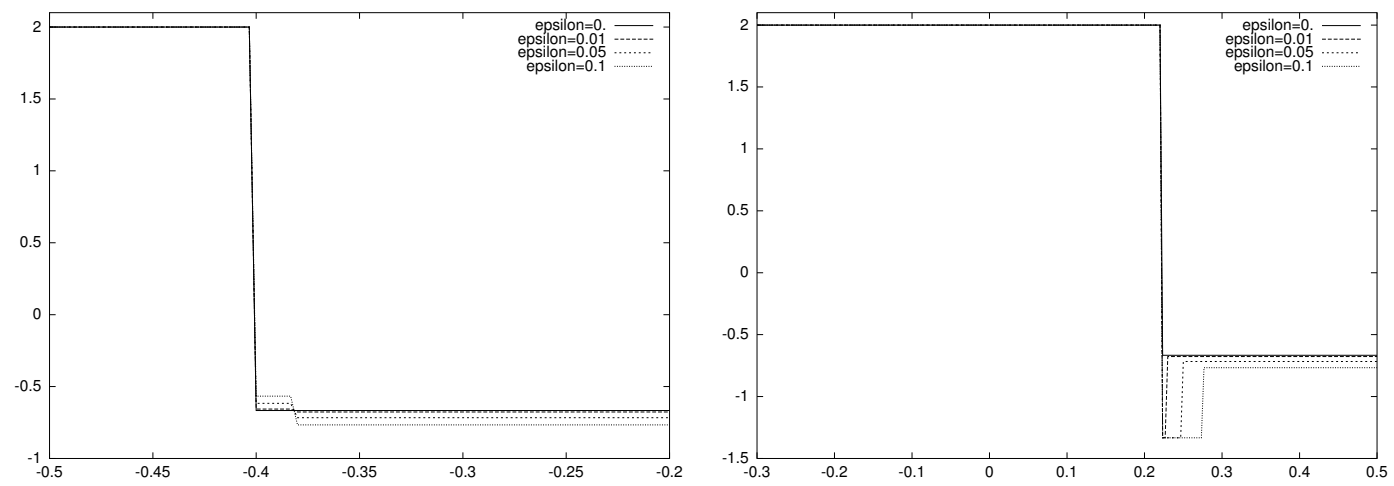

FIG. 3B. Interaction of a classical shock and a rarefaction $(t=0$ and $t=0.2)$.

the solution now contains a two-wave, nonmonotone pattern, precisely a nonclassical shock connecting $u_{L}$ to $\varphi^{\mathrm{b}}\left(u_{L}\right)$ followed by a classical shock connecting $\varphi^{\mathrm{b}}\left(u_{L}\right)$ to $\varphi^{\sharp}\left(u_{L}\right)-\epsilon$.

In Figure 3A, using $\epsilon=0.01, \epsilon=0.05$, and $\epsilon=0.1$ successively, we plotted the numerical Riemann solution for a mesh containing 300 points, at time $t=0.18$, and with $\beta=2 / 3$. We observe that as $\epsilon$ diminishes, the wave speeds of the two shocks become closer and the solution exhibits a spike, while the intermediate value is determined exactly by Glimm's scheme. This feature is not shared by finite difference schemes and the presence of spikes may be an important source of error in numerical computations performed with finite difference schemes.

- Figure 3B: Interaction of a classical shock and a rarefaction. Second, consider the initial data

$$
u_{0}(x)= \begin{cases}u_{L}, & x<-0.40, \\ \varphi^{\sharp}\left(u_{L}\right)+\epsilon, & -0.40<x<-0.38, \\ \varphi^{\sharp}\left(u_{L}\right)-\epsilon, & x>-0.38 .\end{cases}
$$

The solution contains, for small times, a classical shock wave plus a rarefaction wave (with arbitrarily small strength) which interacts in finite time and generates a nonclassical shock. In Figure $3 \mathrm{~B}$, for $\epsilon=0.01, \epsilon=0.05$, and $\epsilon=0.1$ successively, we plot the numerical solution 
obtained with 300 points, at time $t=0.2$ with $\beta=2 / 3$. We note that the total variation of the solution after the interaction is much greater than the total variation of the initial data:

$$
T V(u(t))>T V\left(u_{0}\right) .
$$

- Figure 3C: Large perturbations. This last test is different in nature. We demonstrate here that nonclassical shocks (as well as classical shocks) are stable under large perturbations, in the following sense: when a large perturbation is added at the initial time within a classical shock wave (Figure 3C(I)) or a nonclassical shock wave (Figure 3C(II)), the solution converges asymptotically in time to the unperturbed initial wave. More precisely, this is true up to a possible shift in the location of the discontinuity. The phenomenon is clear for small perturbations, at least: in the nonclassical case, small oscillations on the left-hand side of the nonclassical shock propagate faster than the shock itself, and so pass on its right-hand side in finite time. During the process, the location of the shock is shifted. Next, the oscillations are absorbed by the classical (and then compressive) shock. In the numerical experiments, the amplitude of the perturbation lies between -0.42 et -0.35 . At the time under consideration in Figure 3C(I), the unperturbed shock should be located at the point -0.42 .
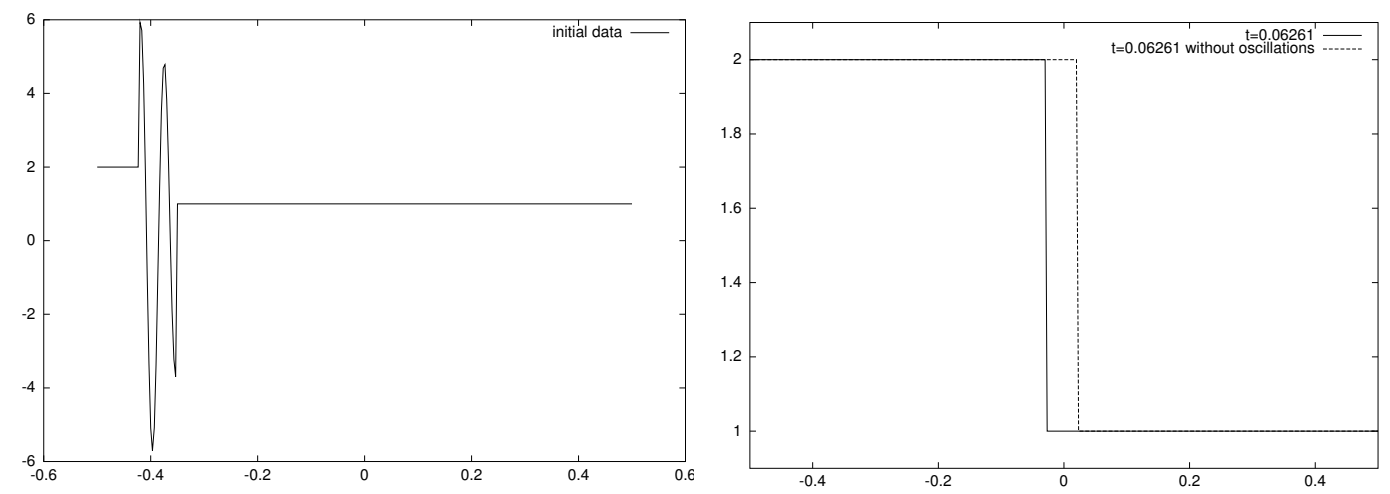

FIG. 3C(I). Large perturbations (classical shock).
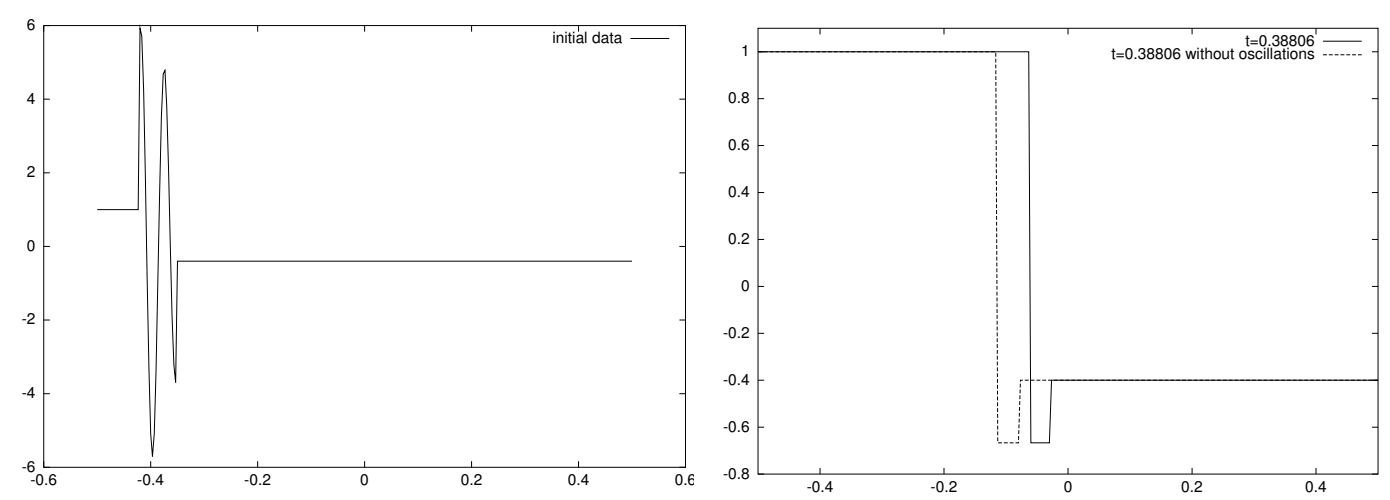

FIG. 3C(II). Large perturbations (nonclassical shock + classical shock). 
Test 4: Periodic data

We begin with the initial condition

$$
u_{0}(x)=-0.5+\sum_{i=0}^{4} \cos (5 i x), \quad x \in[-0.5,0.5],
$$

with periodic boundary conditions

$$
u(-0.5, t)=u(0.5, t) .
$$

The average of the solution over one period is constant and, in our experiments, equals about 0.61826856 . Throughout, we use a mesh containing 300 points.

- Figure 4A: Classical Riemann solution with positive average. To begin with, we use the classical kinetic function corresponding to $\beta=1 / 2$. In Figure 4A, we see that the number of phase transitions is decreasing in time and that the numerical solution converges to a constant value. In the last figure we recognize the so-called $N$-wave which is well known for scalar conservation laws with convex flux. Since the average value is positive, for sufficiently large times the solution takes positive values only, and the convex part of the flux only is relevant.
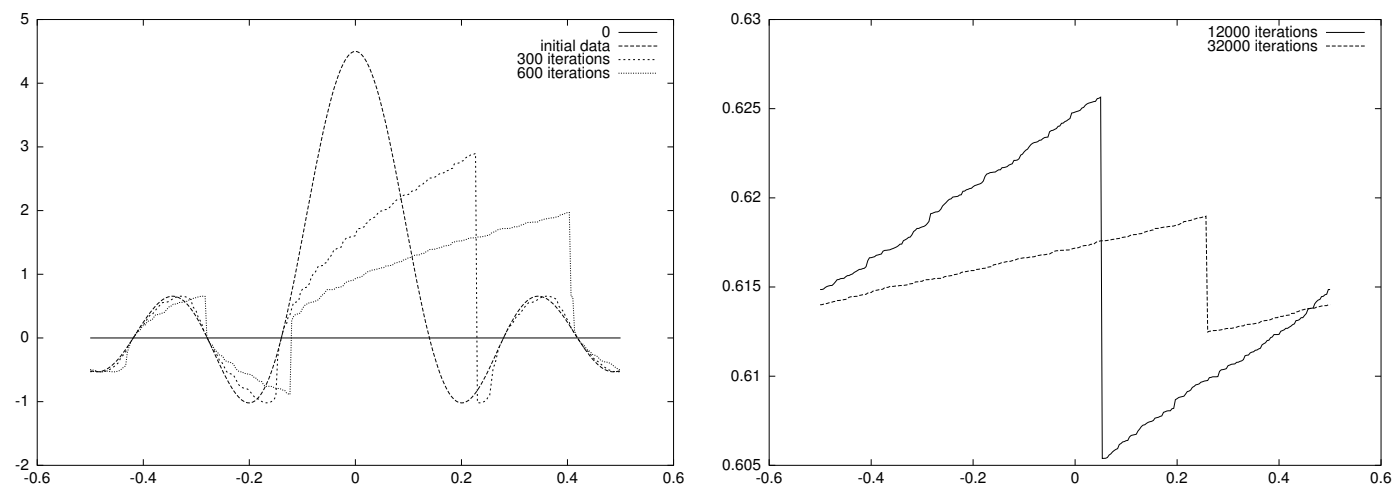

FIG. 4A. Classical Riemann solution with positive average.
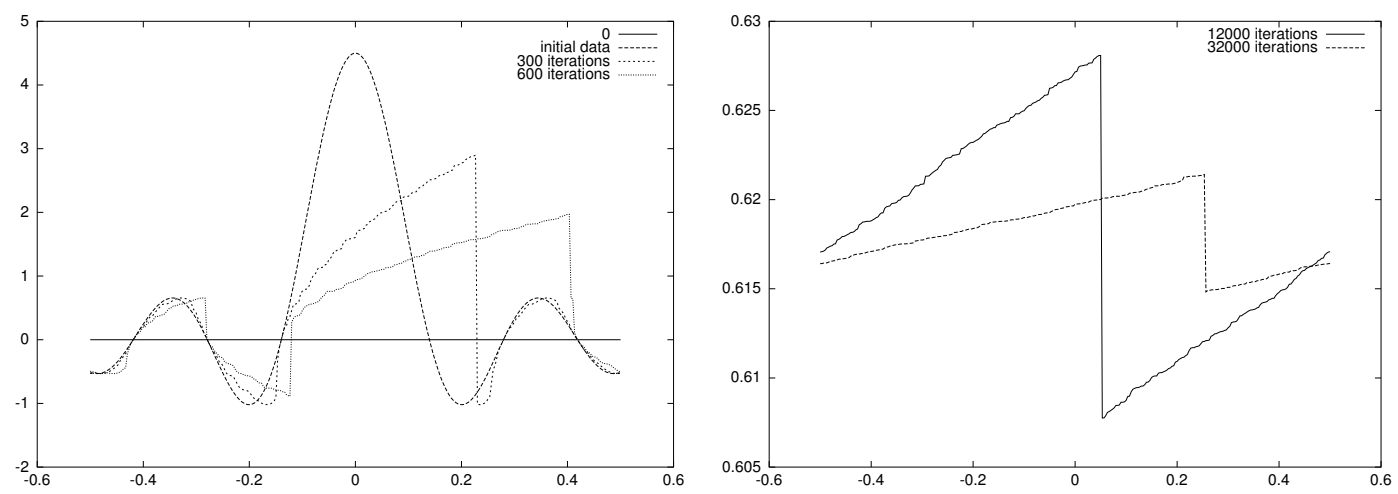

FIG. 4B. Nonclassical Riemann solution with positive average. 

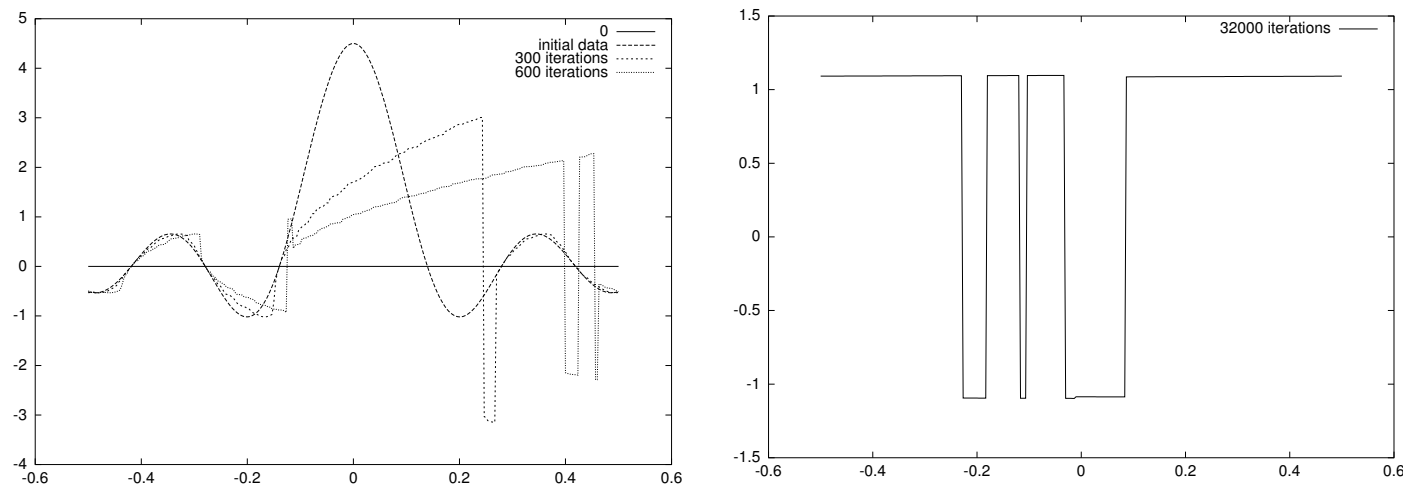

FIG. 4C. Maximally dissipative, nonclassical Riemann solution with positive average.
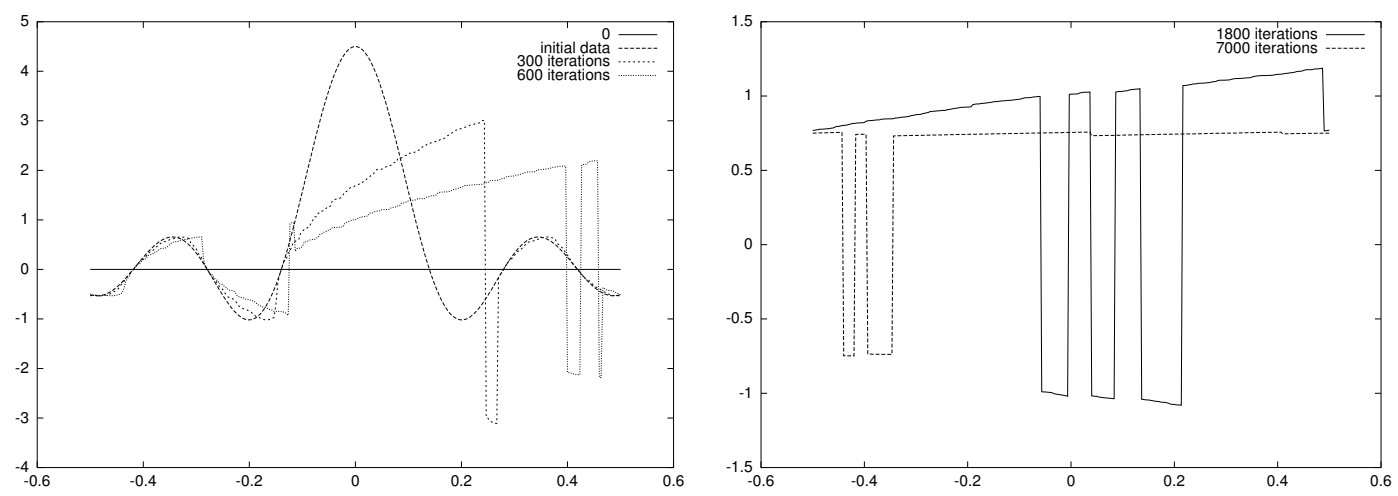

FIG. 4D(I). The case $\beta=1-\epsilon$.
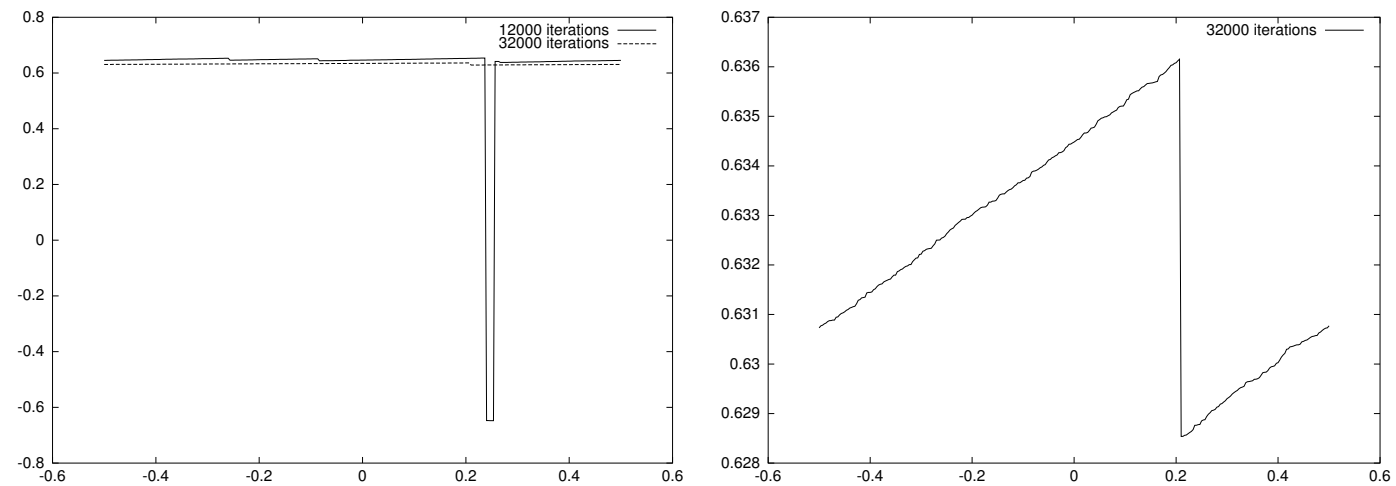

FIG. 4D(II). The case $\beta=1-\epsilon$.

- Figure 4B: Nonclassical Riemann solution with positive average. We now use $\beta=2 / 3$. We observe that the number of phase transitions is still decreasing and that the solution converges to a constant state. Again, we recognize an $\mathrm{N}$-wave. Since the average value is positive, for sufficiently large times the solution takes positive values only, the convex part of the flux only is relevant 

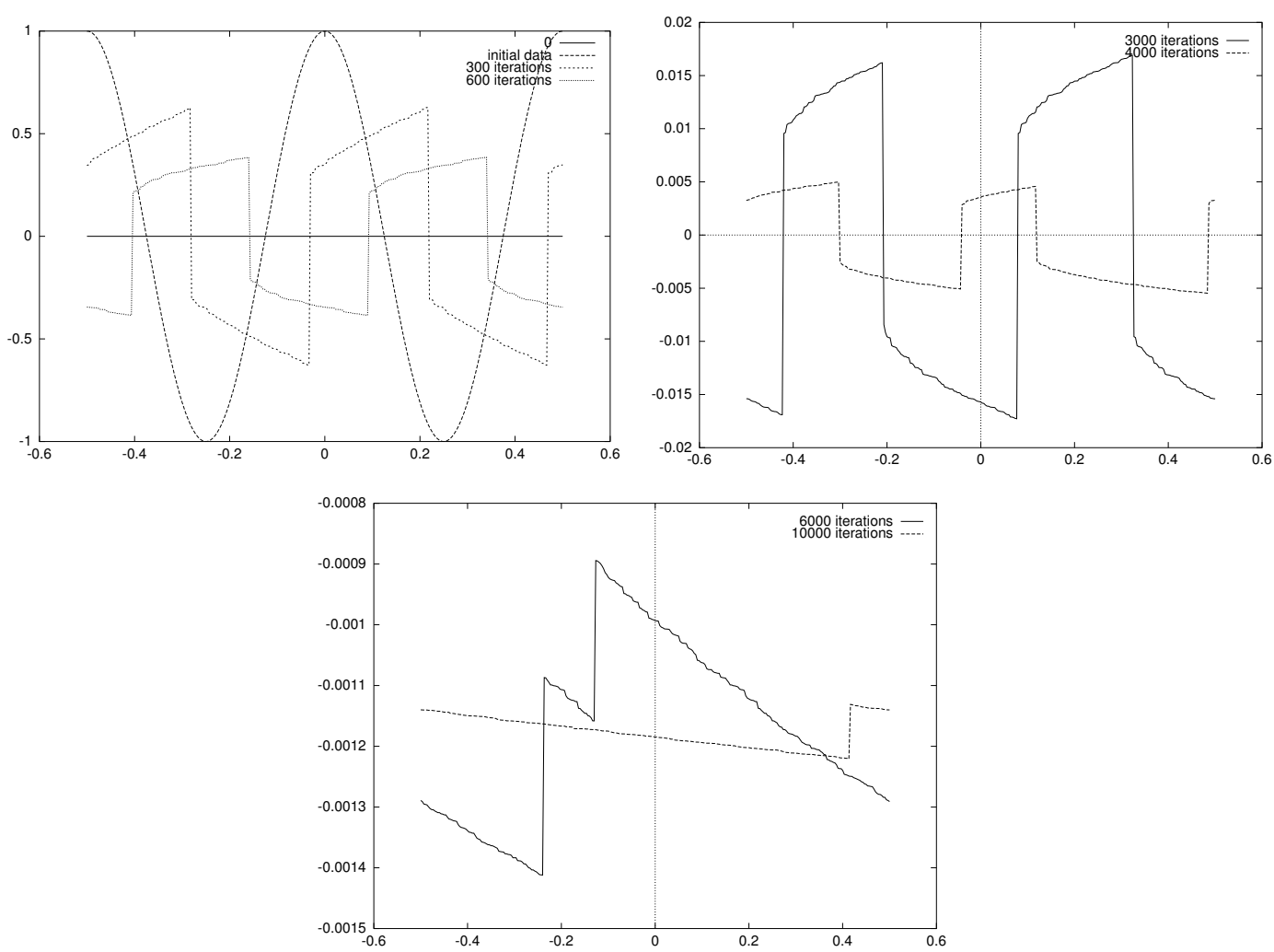

FIG. 4E. Classical Riemann solution with zero average.

and the nonclassical solver coincides with the classical solver in this region, and the solution is eventually entirely classical. The behavior turns out to be very different when the average value under consideration is 0 (see Test 4 below and the following ones). Observe also that Figures 4A and $4 \mathrm{~B}$ are qualitatively similar, the maximal value in the asymptotic nonclassical solution being larger than the one in the classical solution.

- Figure 4C: Maximally dissipative, nonclassical Riemann solution with positive average. Finally, we use $\beta=1$ and observe that the numerical solution no longer converges to a constant state. Instead, the number of phase transitions remains constant and the solution converges to a piecewise constant function made of several phase transitions with coinciding propagation speeds.

- Figure 4D: The case $\beta=1-\epsilon$ with $\epsilon$ small. Taking $\epsilon=0.01$, we observe that the qualitative behavior of the solution is quite analogous to Figure 4B. However, the convergence is very slow, and there is a transient regime during which the solution may appear to behave like the one in Figure 4C. The number of phase transitions is very slowly decreasing but the solution does eventually converge to the average value of the initial data.

- Figure 4E: Classical Riemann solution with zero average. In the remaining tests in this series, we consider the periodic initial condition with period $1 / 2$ :

$$
u_{0}(x)=\cos (4 \pi x)
$$



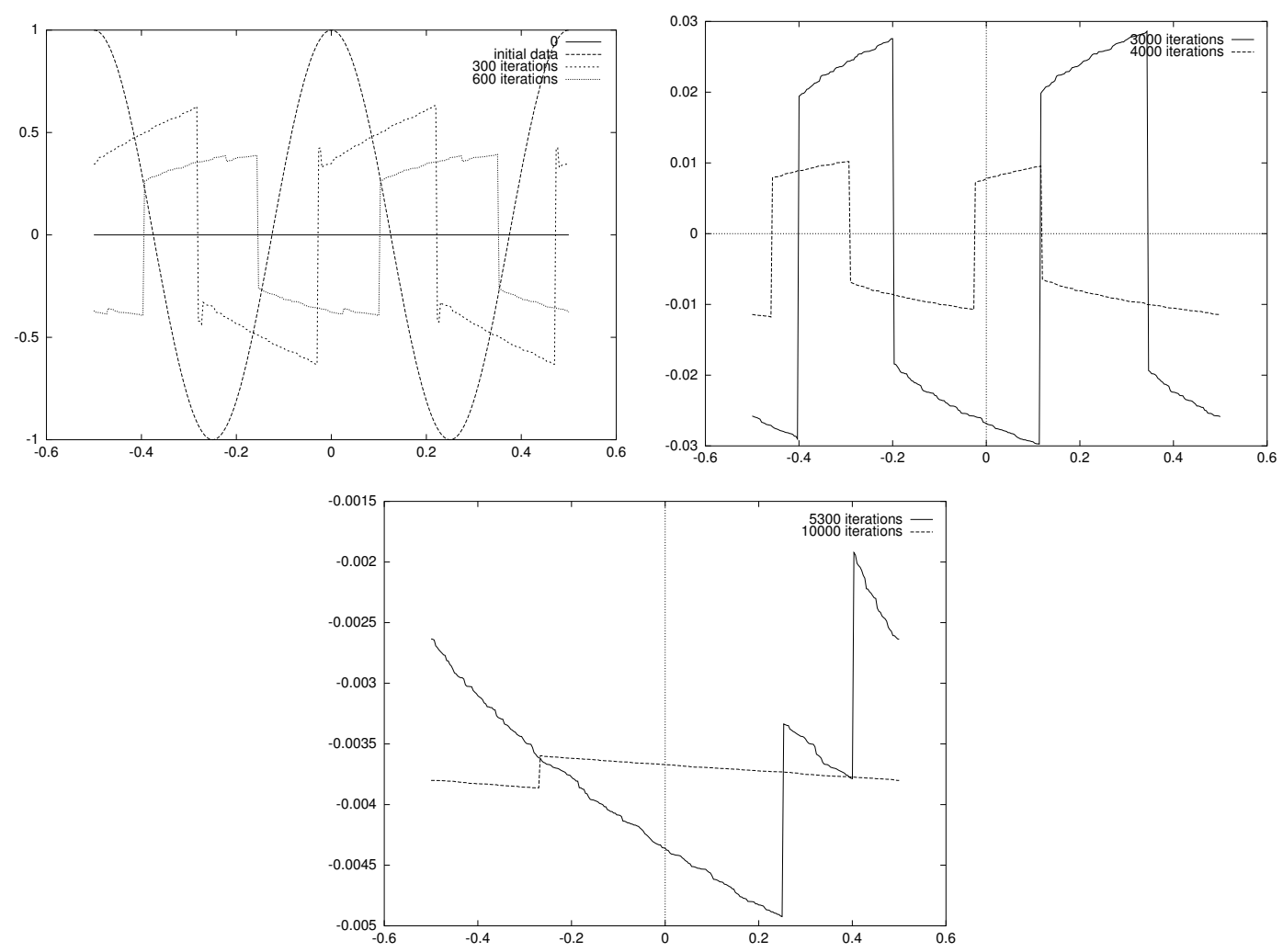

FIG. 4F. Nonclassical Riemann solution with zero average.

which has zero average. This property is the starting point of a new feature in the time-asymptotic behavior we are now highlighting (see also Test 5). Throughout, the mesh contains 300 points and to begin with we take $\beta=1 / 2$. We observe here that the number of phase transitions is decreasing and that the solution converges to zero in a pointwise sense (see mesh refinements below). More precisely, a new feature must be pointed out: there is a transient regime during which the solution exhibits what we will call a "double N-wave pattern", which is somewhat made of two standard well known N-wave profiles with opposite monotonicity. To the best of our knowledge, these double Nwave patterns have never been observed numerically. Note that the period $1 / 2$ is eventually lost as the numerical solution converges in time to a standard $\mathrm{N}$-wave profile. We refer to Test 5 below for further mesh refinements.

- Figure 4F: Nonclassical Riemann solution with zero average. Next, we use a coefficient $\beta$ strictly between $1 / 2$ and 1 , say $\beta=2 / 3$ for instance. The number of phase transitions is decreasing and the solution converges to zero. Again, we observe a double $\mathrm{N}$-wave which eventually disappears and gets transformed into a more familiar single N-wave. See again Test 5 below for a discussion.

- Figure 4G: Maximally dissipative, nonclassical Riemann solution with zero average. Finally, we use $\beta=1$ and notice that the number of phase transitions is now constant in time. The numerical solution converges to a function made of several phase transitions with coinciding propagation speeds. 

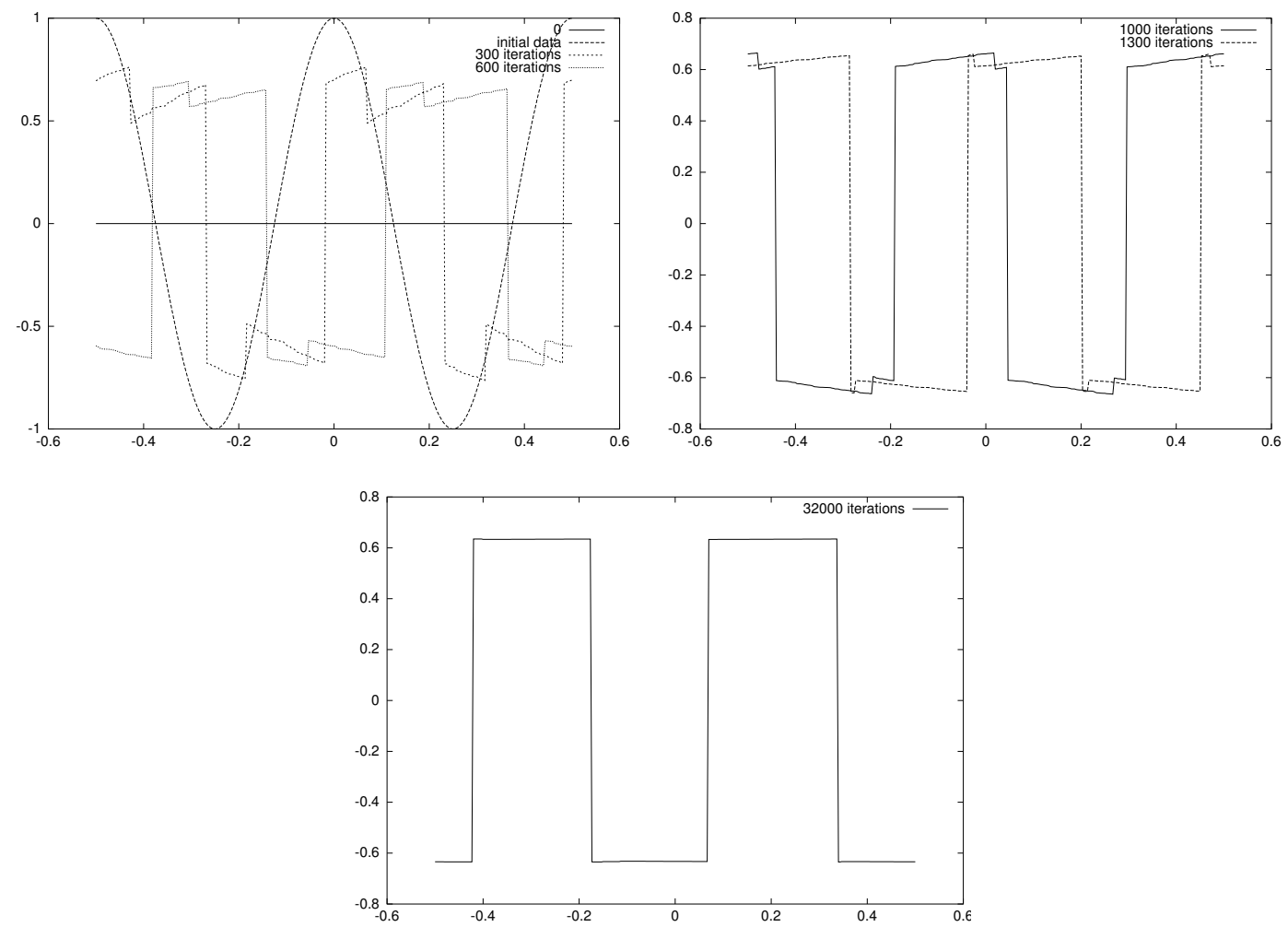

FIG. 4G. Maximally dissipative, nonclassical Riemann solution with zero average.

\section{Test 5: Mesh refinements}

Two mesh refinement experiments are described here. First, using $\beta=2 / 3$ and

$$
u_{0}(x)= \begin{cases}3, & x<-0.375 \\ -3, & x>-0.375\end{cases}
$$

in Figure 5(I) we plotted the numerical solution corresponding to finer and finer mesh lengths, at time $t=0.023$. As expected, the numerical solution approaches the exact solution determined by our nonclassical Riemann solver. This illustrates the convergence property of the random choice scheme to nonclassical entropy solutions.

Second, we plotted in Figure 5(II) the solution with the initial periodic data

$$
u_{0}(x)=\cos (4 \pi x)
$$

for several mesh lengths. As expected, we observe that the numerical solution gets closer to the exact asymptotic solution (that is, 0) pointwise as the mesh is refined. Moreover, we do observe a convergence to the double $\mathrm{N}$-wave profile discovered in Test 4 . Let us mention that the precise asymptotic shape of the exact solution is not known for large values of time $t$, but we conjecture that it is composed of a double $\mathrm{N}$-wave. It would be very interesting to check this conjecture from the 

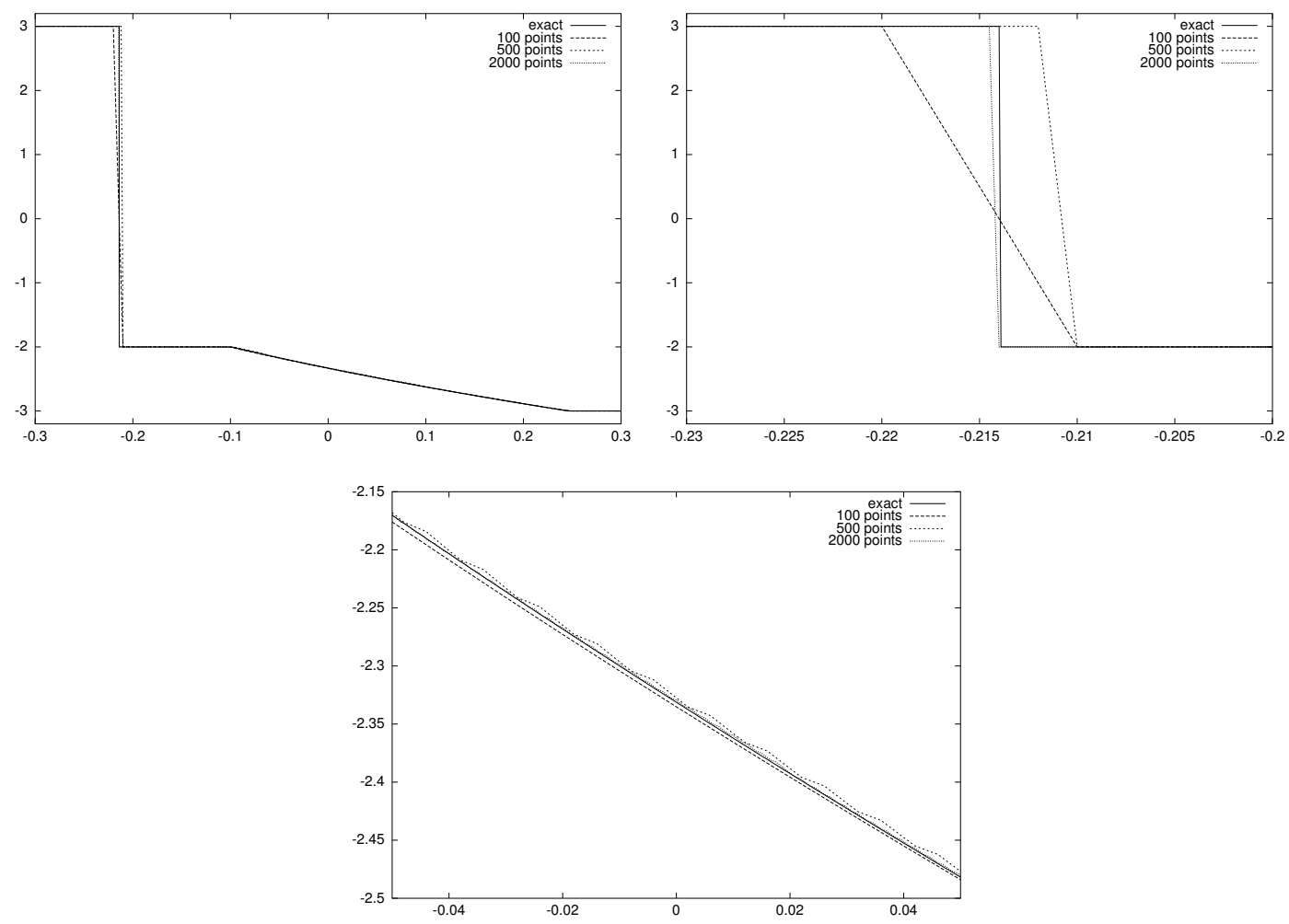

FIG. 5(I). Mesh refinement (global solution and zooms).

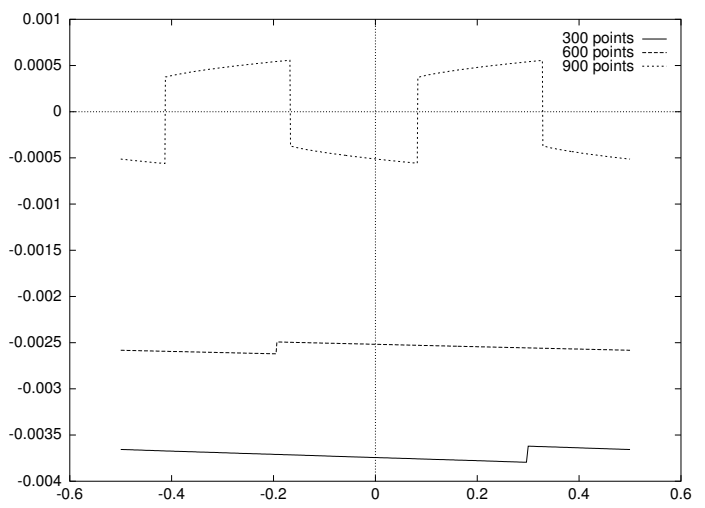

FIG. 5(II). Mesh refinement (periodic data).

analytical standpoint. To conclude this test, note that the averaged mass of the two lower solutions in Figure 5(II) is not zero: this is due to the fact that Glimm's scheme is not conservative! Of course, this also explains the fact that for very large times the double $\mathrm{N}$-wave patterns eventually disappear in the previous simulations. 


\section{Hyperbolic model of nonlinear elastodynamics}

We now turn to the study of the extension of the properties we have pointed out in the previous section to a model of nonlinear elastodynamics. We consider the following model of two conservation laws for the mass and momentum of some nonlinear material, with prescribed initial data $\left(v_{0}, w_{0}\right)$ :

$$
\begin{aligned}
& \partial_{t} v-\partial_{x} \sigma(w)=0, \quad x \in \mathbb{R}, t>0, \\
& \partial_{t} w-\partial_{x} v=0, \\
& (v(x, 0), w(x, 0))=\left(v_{0}(x), w_{0}(x)\right), \quad x \in \mathbb{R},
\end{aligned}
$$

where $v$ and $w$ represent the velocity and the deformation gradient of the material, respectively. The strain-stress function $\sigma$ is taken to be

$$
\sigma(w)=w^{3}+m w,
$$

with $m=2$ for the numerical experiments below (except when mentioned otherwise). The Cauchy problem 3.1 is supplemented with the following kinetic function for each undercompressive nonclassical shock:

$$
\varphi^{\beta}(w)=-\beta w,
$$

where $\beta \in[1 / 2,1)$ is a parameter. We refer to [24] for a detailed discussion of the nonclassical Riemann solver corresponding to this problem. We simply recall here that when $\beta=1 / 2$ the nonclassical Riemann solver actually coincides with the classical solver characterized, for instance, by the Liu entropy criterion. When $\beta>1 / 2$, the Riemann solution may contain shock waves which violate the Liu criterion, are undercompressive, and satisfy the kinetic relation

$$
\begin{array}{ll}
w_{+}=\varphi^{\beta}\left(w_{-}\right) & \text {for shock with positive speed } \\
w_{-}=\varphi^{\beta}\left(w_{+}\right) & \text {for shock with negative speed. }
\end{array}
$$

As was noticed for scalar conservation laws, the maximally dissipative kinetic relation corresponding to $\beta=1$ is not strictly allowed in the general theory (see [21]) but can be regarded as an interesting limiting case. The corresponding shock waves have zero-entropy dissipation and achieve the largest propagation speed (in modulus) among all admissible nonclassical shocks.

Test 6: Splitting of shock waves

- Figure 6A: Small perturbations of a classical shock. We consider the Riemann initial data

$$
\left(v_{0}(x), w_{0}(x)\right)= \begin{cases}(1,1+\epsilon), & x<0.5, \\ (-11,-3), & x>0.5,\end{cases}
$$

and we plot the corresponding numerical solution for several values of $\epsilon$. When $\epsilon=0$, the exact solution of the Riemann problem is a single classical shock. We see that the structure for arbitrary small values $\epsilon$ is entirely different from the one with $\epsilon=0$. In particular, the $L^{\infty}$-norm of the solution is not continuous as $\epsilon \rightarrow 0$. We use a mesh with 600 points by unit interval, $t=0.4$, and $\beta=2 / 3$. 

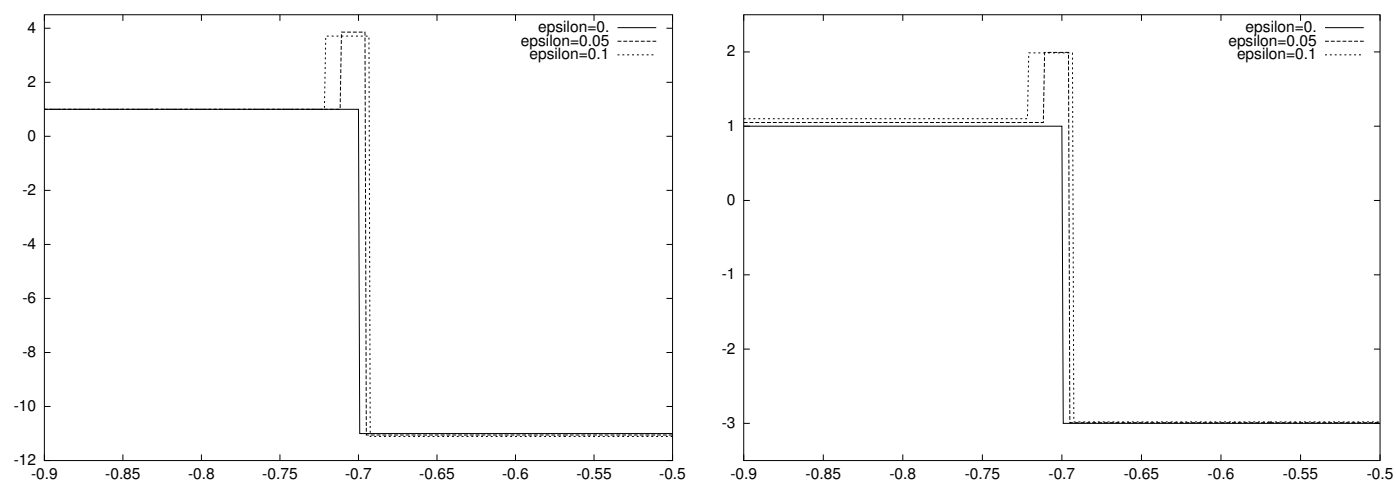

FIG. 6A. Small perturbations of a classical shock ( $v$ and $w$ components).
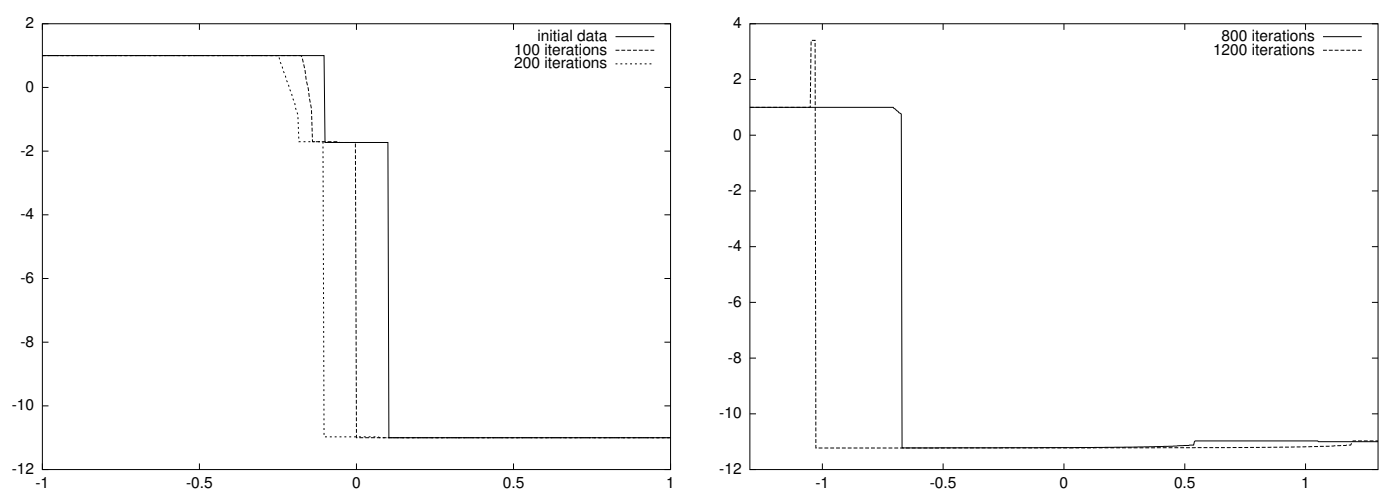

FIG. 6B(I). Interaction of two classical shocks ( $v$ component).
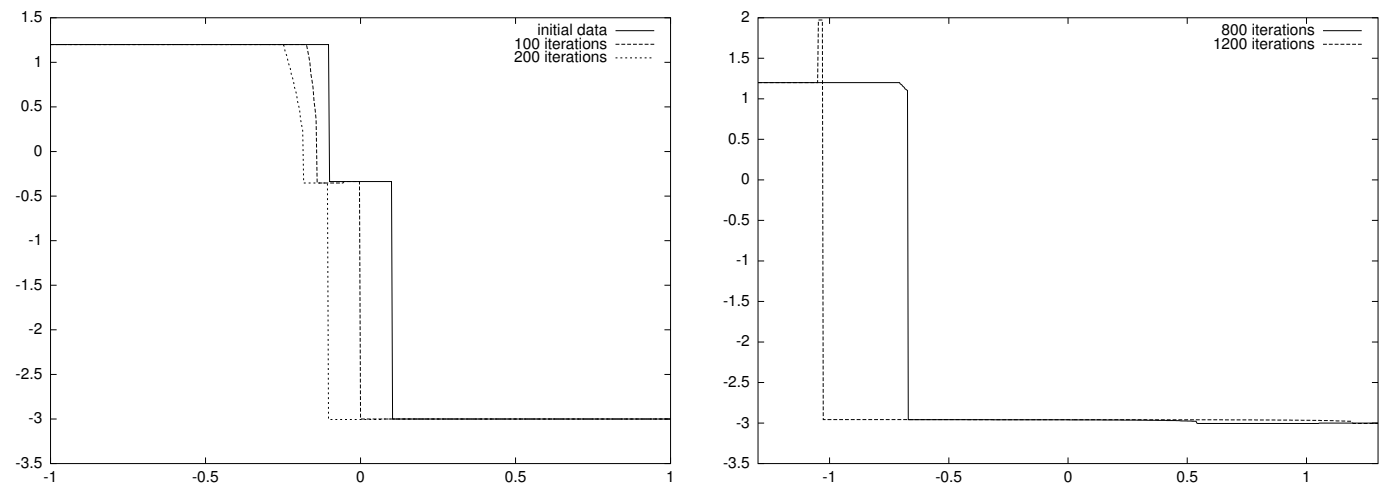

FIG. 6B(II). Interaction of two classical shocks ( $w$ component).

- Figure 6B: Interaction of two classical waves. As in the scalar case, two incoming waves (of the same family) may interact and can generate a nonclassical shock wave. The total variation of the solution increases at the interaction and the $w$ component becomes nonmonotone after the interaction. Of course, it is well known that for hyperbolic systems of conservation laws, the total 
variation may increase at interactions; however, the increase here is not of the order of the product of the strengths of the incoming waves. Specifically, we consider the initial data

$$
\left(v_{0}(x), w_{0}(x)\right)= \begin{cases}(1,1.2), & x<-0.1 \\ (-1.72814176,-0.337385342), & -0.1<x<0.1 \\ (-11,-3), & x>0.1\end{cases}
$$

and we plot the solutions after various iteration numbers. We use a mesh with 300 points per unit interval.

- Figure 6C: Large perturbations. Finally, we observe that the nonclassical Riemann solver (as well as the classical one) is stable under possibly large perturbations. Oscillations ranging between -0.03 and 0 are introduced in a given initial data. The discontinuity is located at 0 when no oscillations are present. The left-hand state is taken to be $\left(v_{L}, w_{L}\right)=(0,1)$ and the right-hand state is $\left(v_{R}, w_{R}\right)=(-6.71190015066,-2)$. We use a mesh containing 200 points per unit interval. We observe that the unperturbed profile is recovered asymptotically in time, but the position of the discontinuity is shifted.
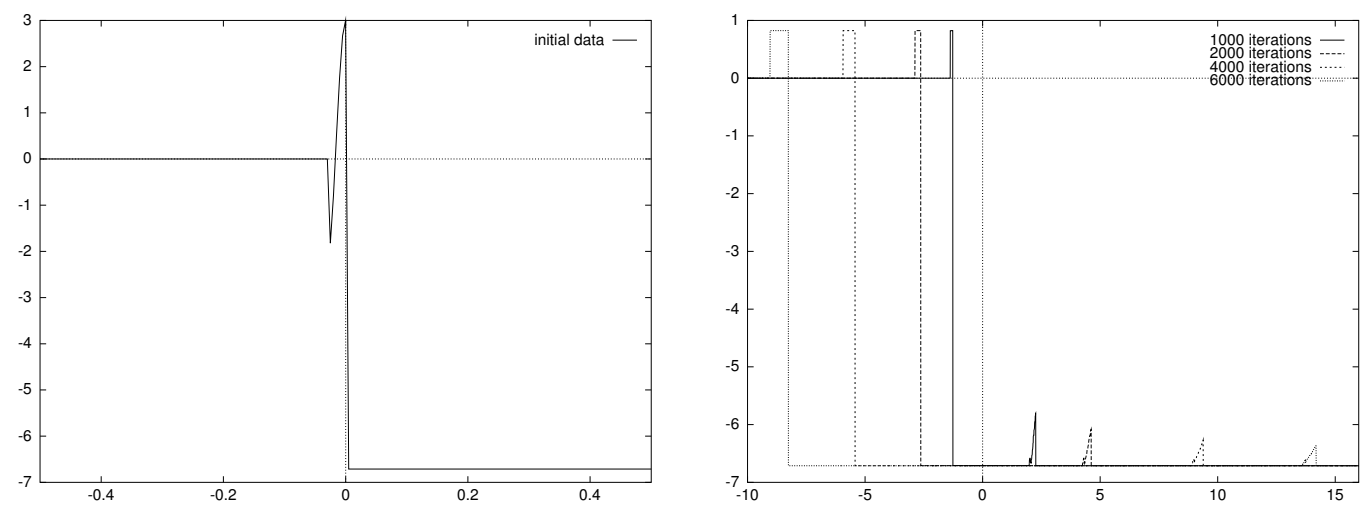

FIG. 6C(I). Large perturbations ( $v$ component).
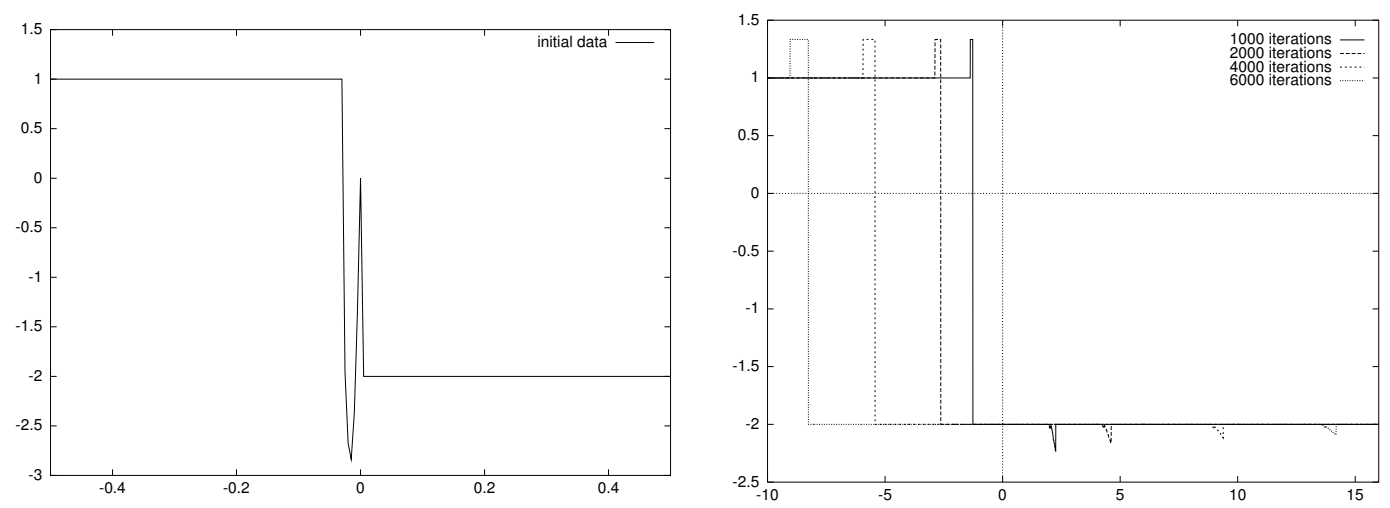

FIG. 6C(II). Large perturbations ( $w$ component). 


\section{Test 7: Periodic data}

As in the scalar case, we now study the asymptotic behavior of the solutions for several kinetic functions. We use the stress function given by (3.2), but now with $m=0.05$, and a mesh containing 1000 points.

- Figure 7A: The classical case. First, we consider the case $\beta=1 / 2$ and the following periodic initial data:

$$
\left(v_{0}(x), w_{0}(x)\right)= \begin{cases}(0,0.4), & x<x_{0}, \\ (0,-0.2), & x_{0}<x<x_{1}, \\ (0,0.4), & x>x_{1},\end{cases}
$$

where $x_{0}$ and $x_{1}$ will be specified shortly. Each of the following computations is performed over the unit interval $[-0.5,0.5]$ with periodic boundary conditions.

First, we choose $x_{0}=0$ and $x_{1}=0.3$ so that the corresponding average $\bar{w}_{0}$ of $w_{0}$ is positive, precisely $\bar{w}_{0}=0.22$. The $v$ and $w$ components are plotted in Figures 7A(I) and 7A(II), while Figure 7A(III) shows the two Riemann invariants

$$
v-\int^{w} \sqrt{\sigma^{\prime}(x)} d x \text { and } v+\int^{w} \sqrt{\sigma^{\prime}(x)} d x
$$

after linearization near the average value $\bar{w}_{0}$, that is,

$$
v-\sqrt{\sigma^{\prime}\left(\bar{w}_{0}\right)} w \text { and } v+\sqrt{\sigma^{\prime}\left(\bar{w}_{0}\right)} w .
$$

We observe that as the time evolves, these variables exhibit two standard $N$-waves, propagating at speed with opposite signs, as is well known from the general theory of genuinely nonlinear systems.

Second, we choose $x_{0}=-0.3$ and $x_{1}=-0.3+2 / 3$, so that the averages of $v_{0}$ and $w_{0}$ are both 0 . Figures $7 \mathrm{~A}(\mathrm{IV})$ and $7 \mathrm{~A}(\mathrm{~V})$ show the $v$ and $w$ components, respectively. The Riemann invariants linearized near 0 , that is,

$$
v-\sqrt{\sigma^{\prime}(0)} w \text { and } v+\sqrt{\sigma^{\prime}(0)} w,
$$

exhibit a double $N$-wave pattern; see Figure $7 \mathrm{~A}(\mathrm{VI})$. We recall that the same behavior of the conservative variable was observed in the scalar case. (Compare with Figures 4E, 4F and 5(II).) Recovering this new feature for a system of conservation laws is an interesting issue.

- Figure 7B: The nonclassical case. We now study the nonclassical value $\beta=3 / 4$ together with the following periodic initial data:

$$
\left(v_{0}(x), w_{0}(x)\right)= \begin{cases}(0.1,0.4), & x<x_{0}, \\ (0,-0.2), & x_{0}<x<x_{1}, \\ (0.1,0.4), & x>x_{1} .\end{cases}
$$

Figures $7 \mathrm{~B}(\mathrm{I}), 7 \mathrm{~B}(\mathrm{II})$, and $7 \mathrm{~B}(\mathrm{III})$ correspond to the choice $x_{0}=0$ and $x_{1}=3$ (so that the average of $w_{0}$ is 0.22 ), while Figures $7 \mathrm{~B}(\mathrm{IV}), 7 \mathrm{~B}(\mathrm{~V})$, and $7 \mathrm{~B}(\mathrm{VI})$ show the case $x_{0}=-0.3$ and $x_{1}=-0.3-$ $2 / 3$ for which the average of $w_{0}$ equals zero. The same asymptotic behaviors as in the classical case are observed for the linearized Riemann invariants, that is, standard $N$-waves (Figure $7 \mathrm{~B}(\mathrm{III})$ ) and double $N$-waves (Figure $7 \mathrm{~B}(\mathrm{VI})$ ), depending on the average of $w_{0}$. 

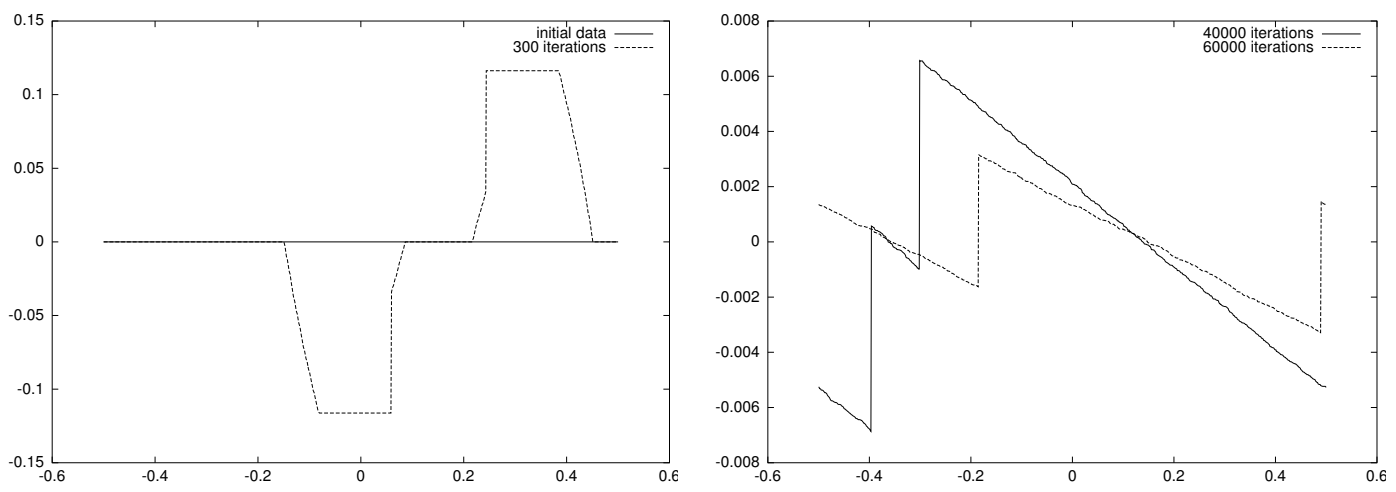

FIG. 7A(I). Classical Riemann solution ( $v$ component).
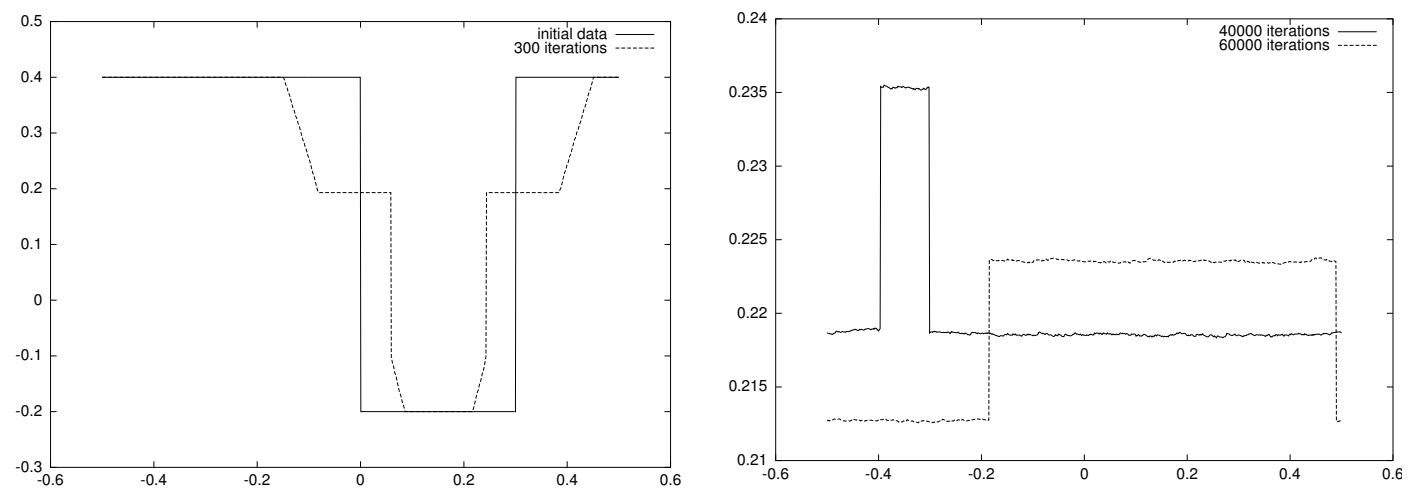

FIG. 7A(II). Classical Riemann solution ( $w$ component).
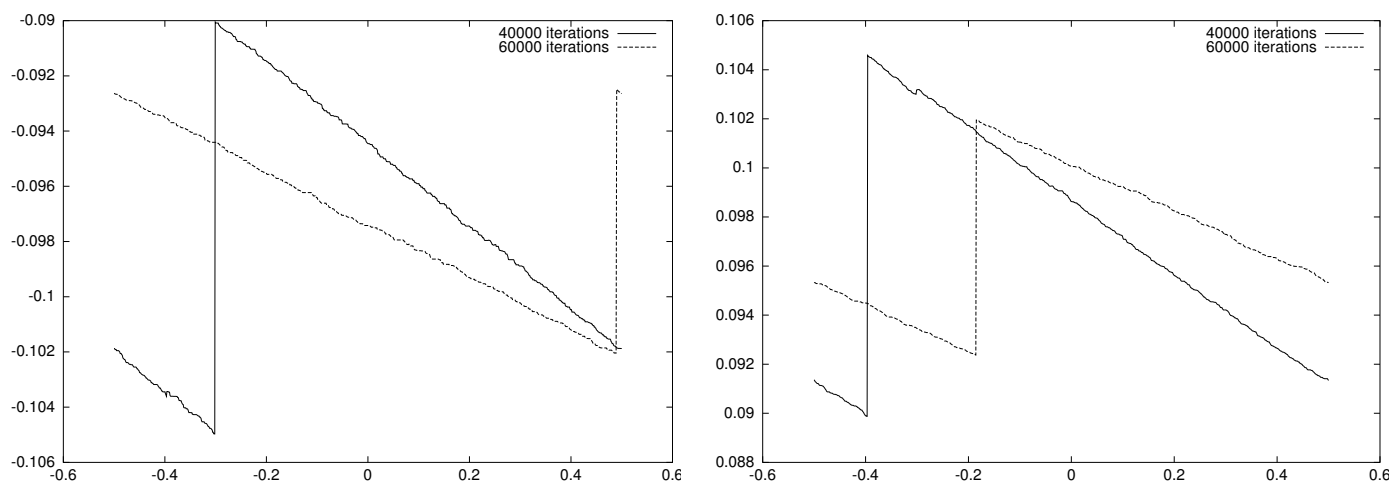

FIG. 7A(III). Classical Riemann solution. (Linearized Riemann invariants.). 

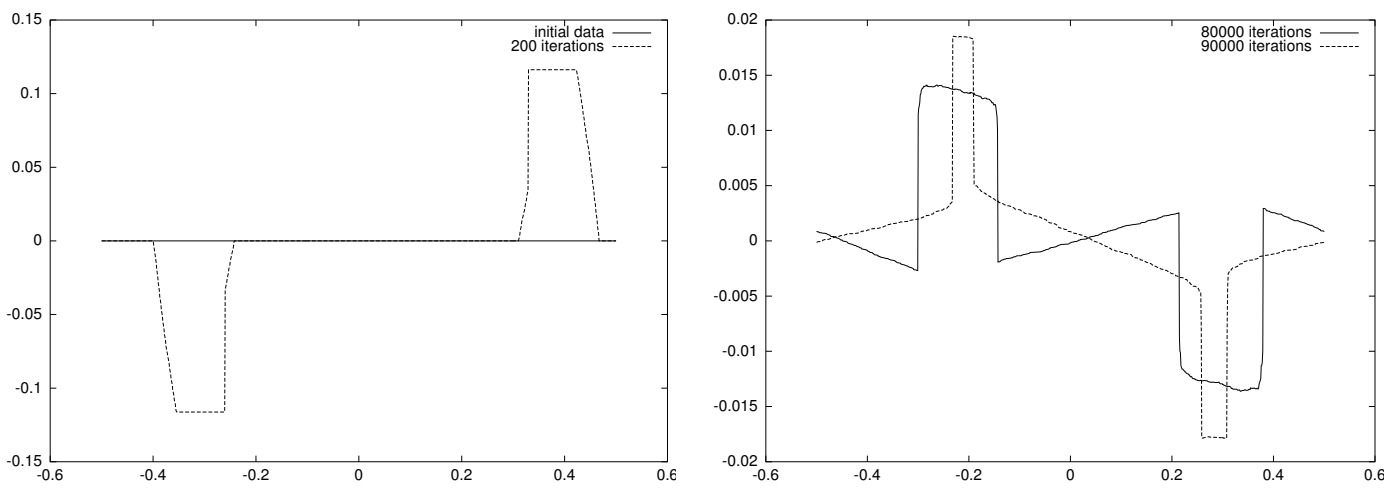

FIG. 7A(IV). Classical Riemann solution ( $v$ component).
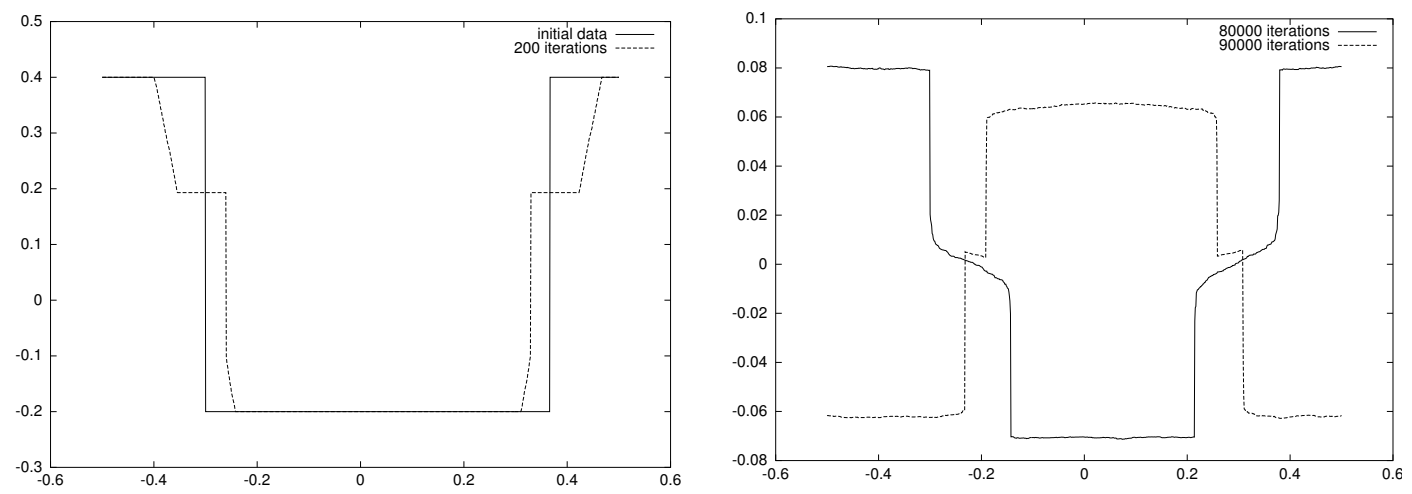

FIG. 7A(V). Classical Riemann solution ( $w$ component).
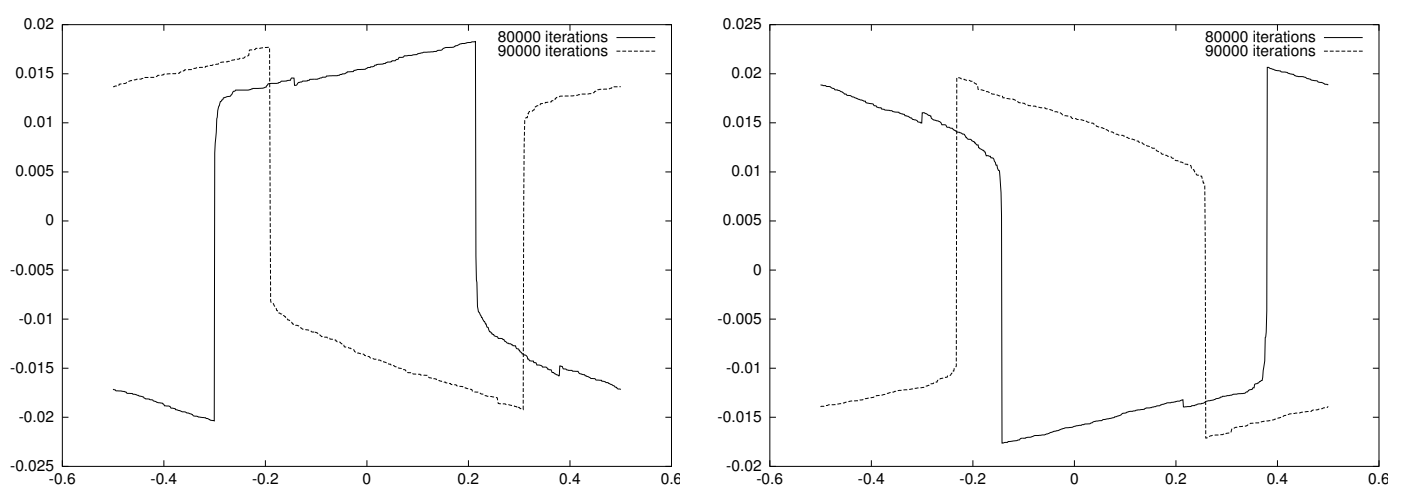

FIG. 7A(VI). Classical Riemann solution. (Linearized Riemann invariants.). 

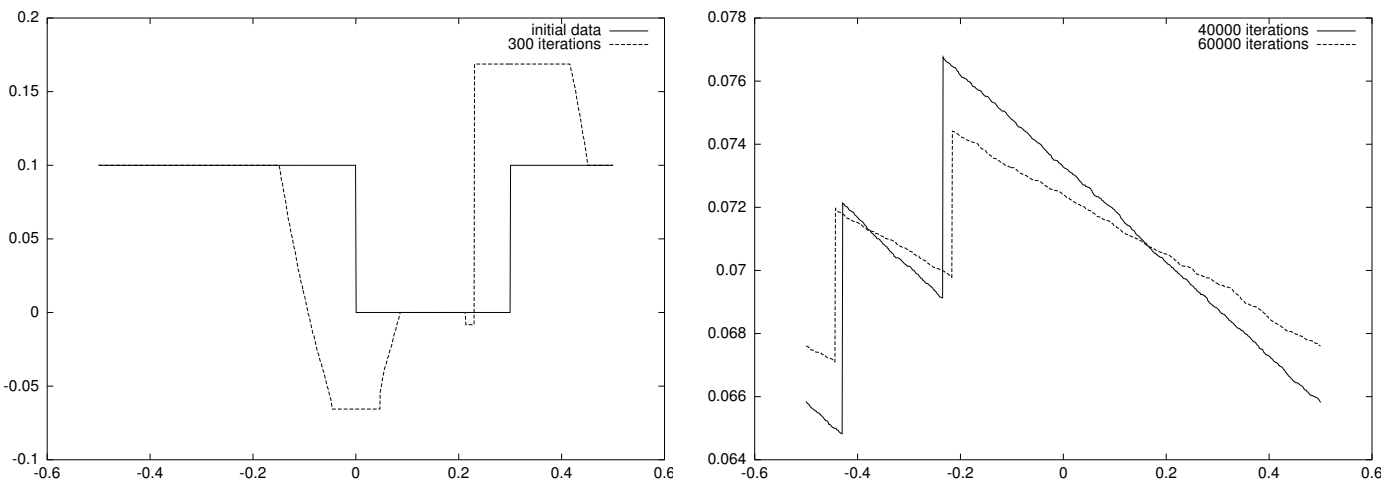

FIG. 7B(I). Nonclassical Riemann solution ( $v$ component).
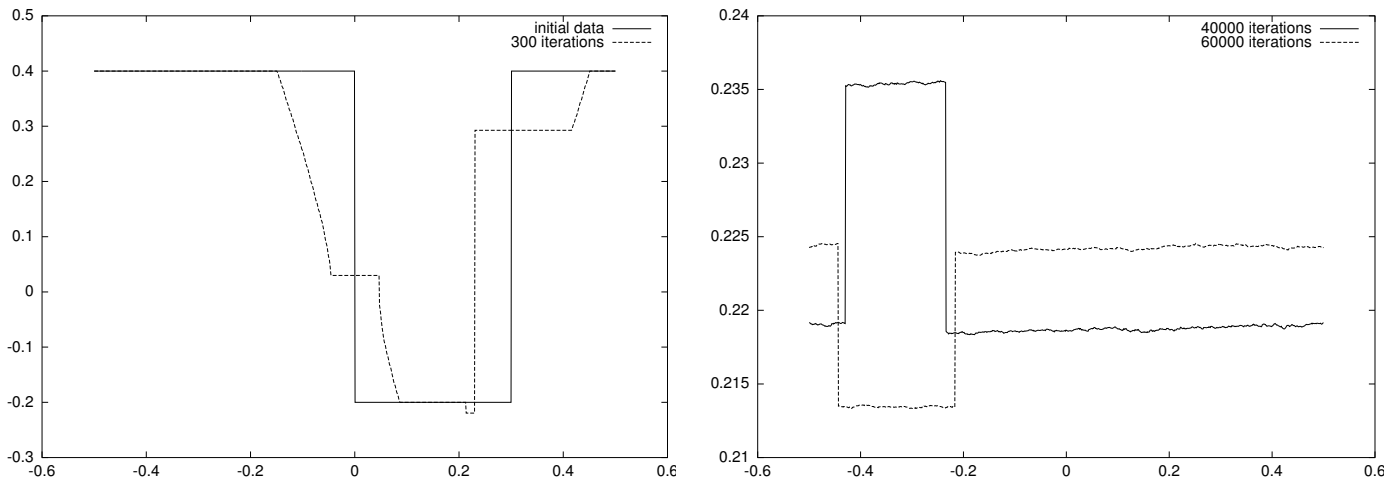

FIG. 7B(II). Nonclassical Riemann solution ( $w$ component).
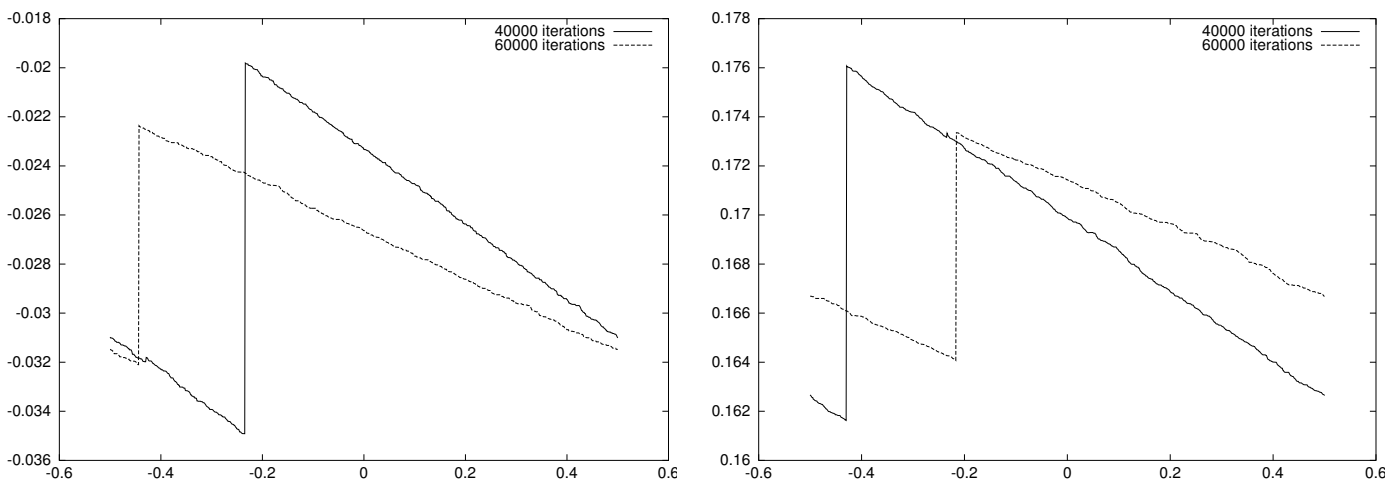

FIG. 7B(III). Nonclassical Riemann solution (Linearized Riemann invariants). 

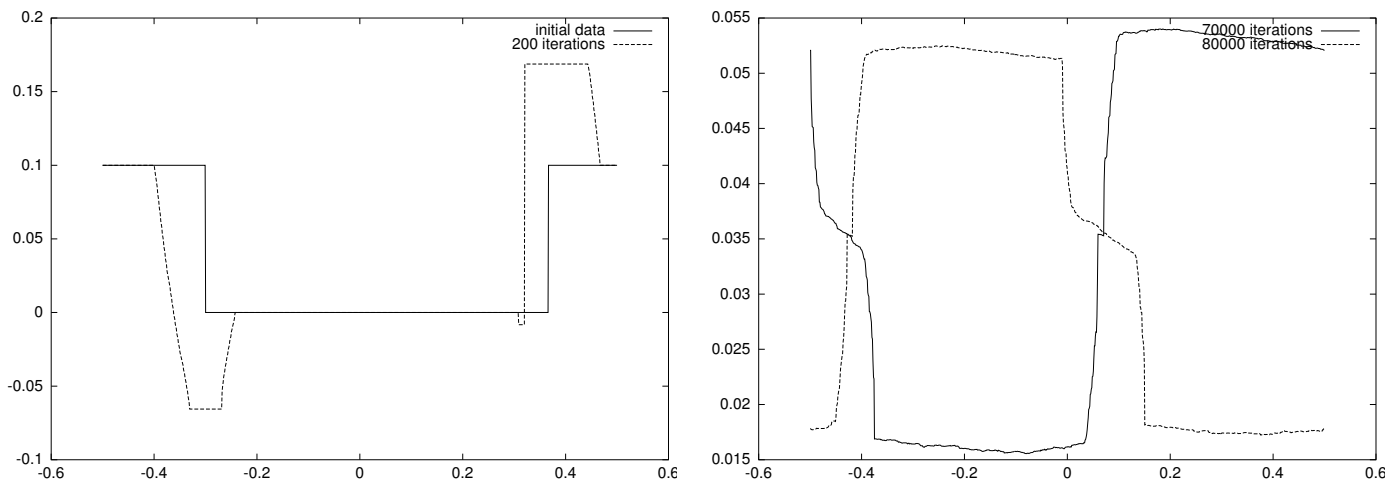

FIG. 7B(IV). Nonclassical Riemann solution ( $v$ component).
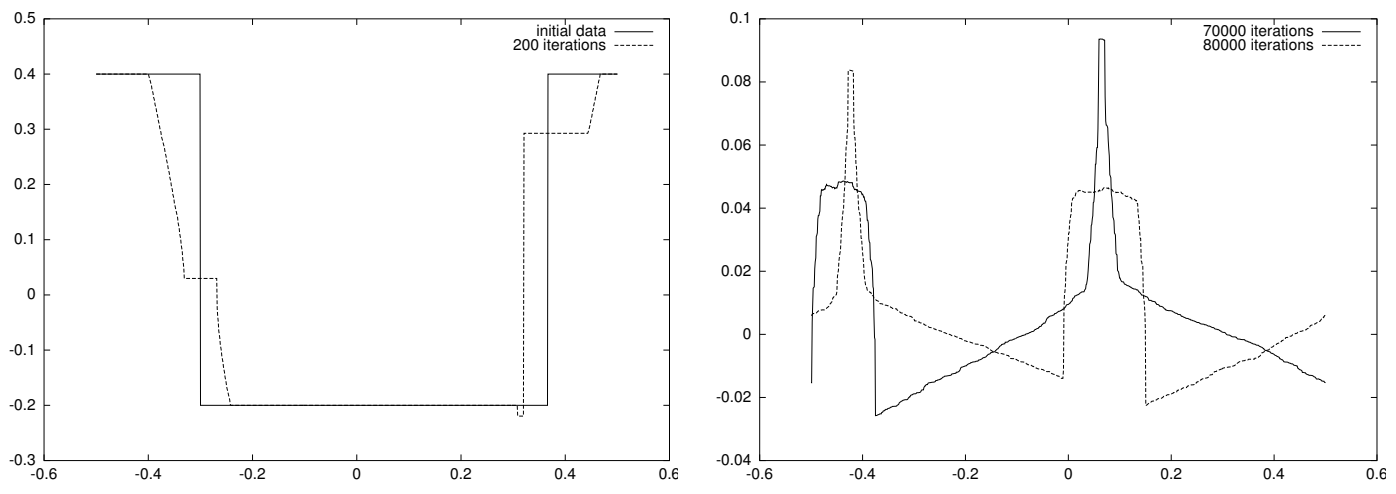

FIG. 7B(V). Nonclassical Riemann solution ( $w$ component).
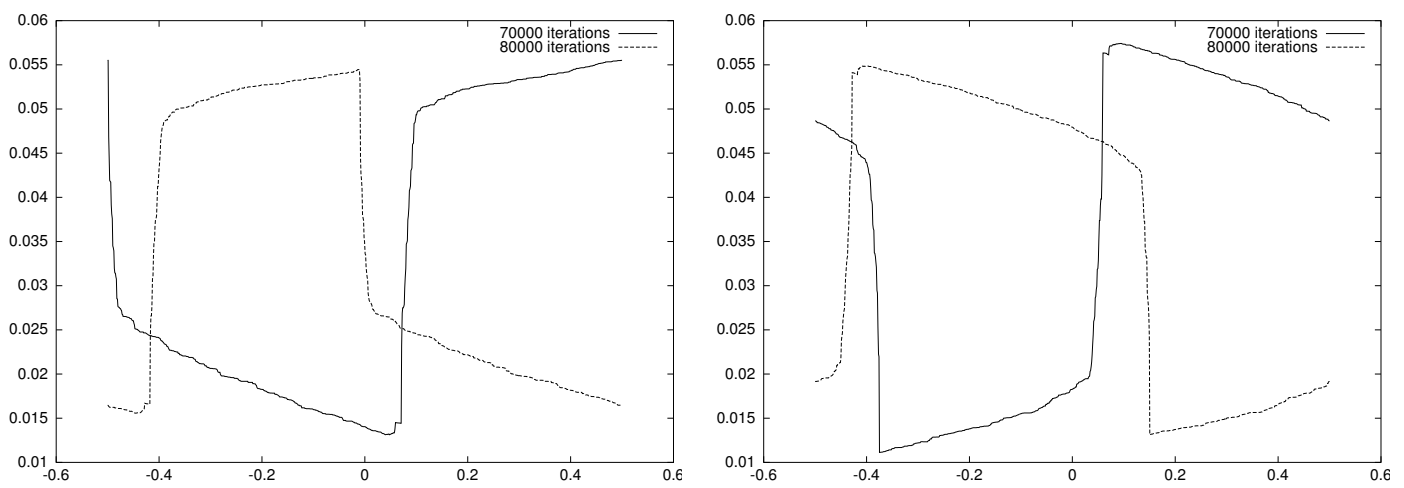

FIG. 7B(VI). Nonclassical Riemann solution (Linearized Riemann invariants). 
- Figure 7C: The maximally dissipative nonclassical case. Here we investigate the choice $\beta=1$, with the following periodic initial data:

$$
\left(v_{0}(x), w_{0}(x)\right)= \begin{cases}(-0.3,0.4), & x<-0.3, \\ (0,-0.2), & -0.3<x<-0.3+2 / 3, \\ (-0.3,0.4), & x>-0.3+2 / 3 .\end{cases}
$$

Here, the average of the $w$ component equals zero. Figure 7C(I) shows the component $w$ after various numbers of iterations. For large times, we observe (as in the scalar case) that the solution tends to a piecewise constant function made of two phase transitions satisfying the prescribed kinetic relation. Since $\beta=1$, they have the same propagation speed. Observe also that the number of phase transitions is equal to the number of phase transitions in the initial data.

It is worth pointing out that another qualitative behavior can be observed with the maximally dissipative solver. Indeed, if we consider the periodic initial data

$$
\left(v_{0}(x), w_{0}(x)\right)= \begin{cases}(0,0.4), & x<-0.3, \\ (0,-0.2), & -0.3<x<-0.3+2 / 3, \\ (0,0.4), & x>-0.3+2 / 3,\end{cases}
$$
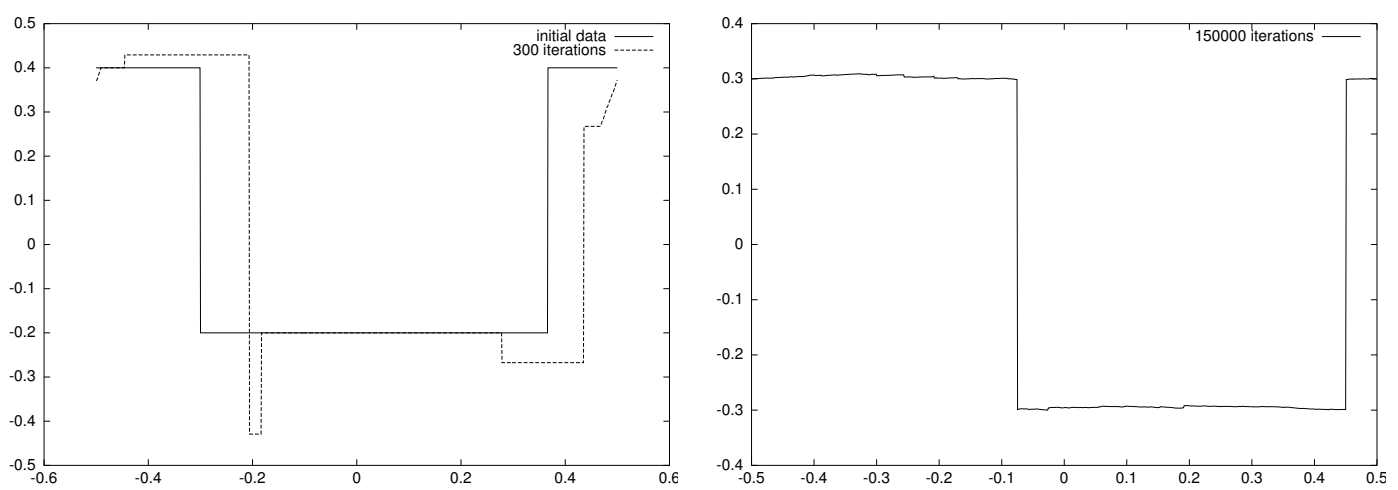

FIG. 7C(I). Maximally dissipative, nonclassical Riemann solution ( $w$ component).
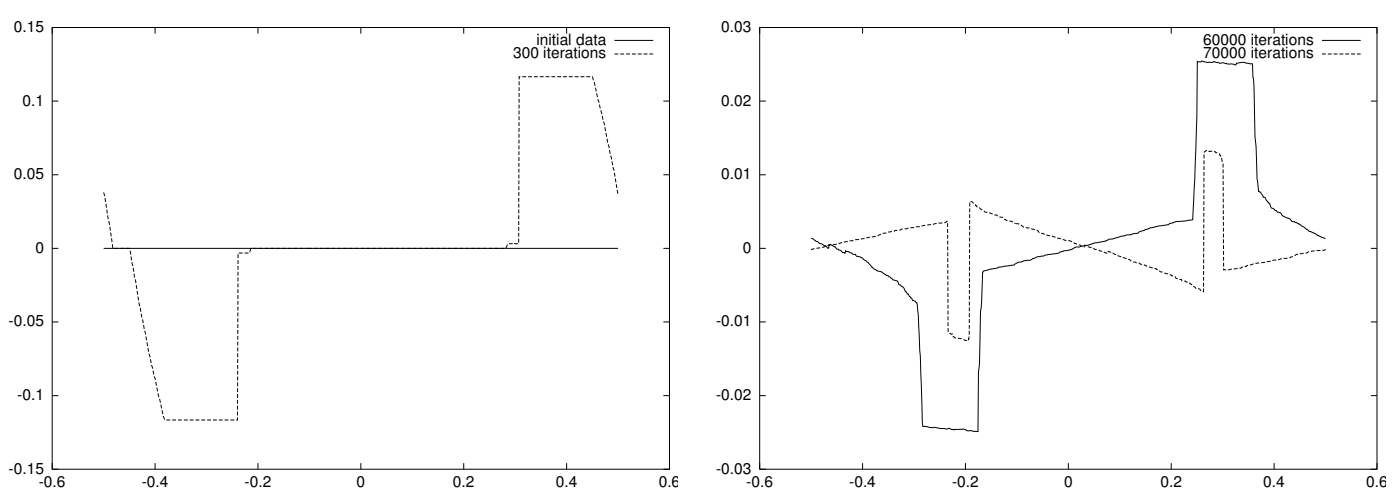

FIG. 7C(II). Maximally dissipative Riemann solution ( $v$ component). 

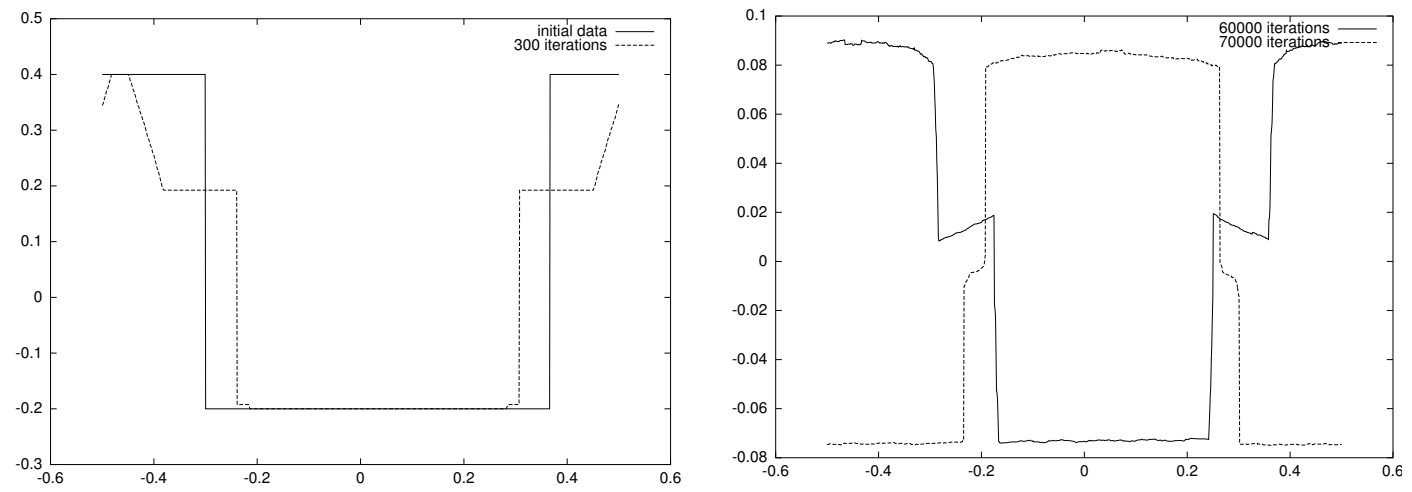

FIG. 7C(III). Maximally dissipative Riemann solution ( $w$ component).
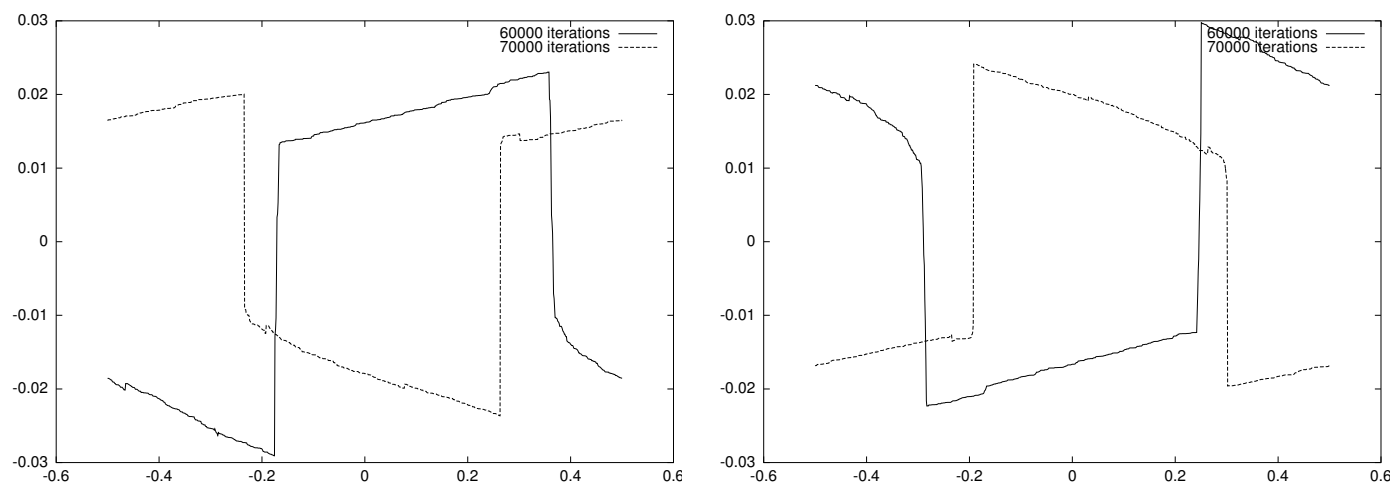

FIG. 7C(IV). Maximally dissipative Riemann solution. (Linearized Riemann invariants.).
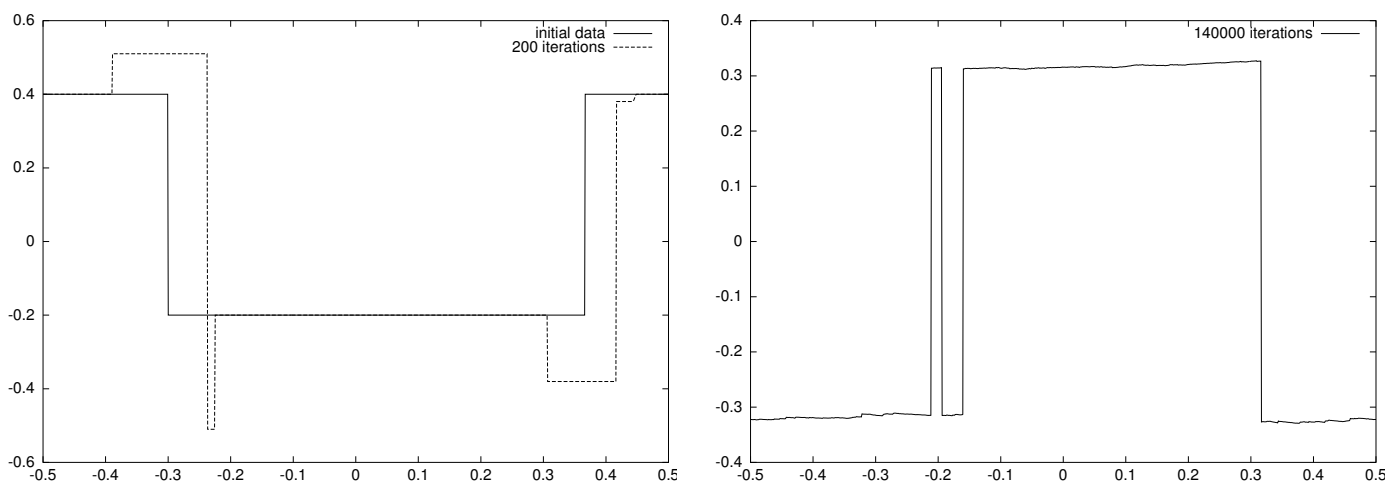

FIG. 7C(V). Increasing the number of phase transitions ( $w$ component). 

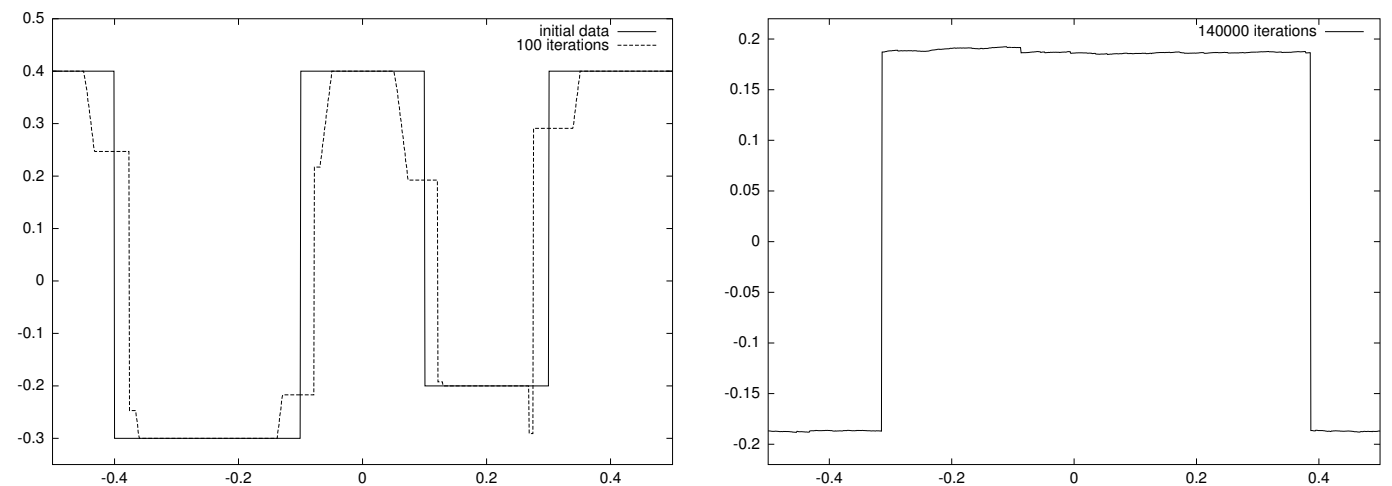

FIG. 7C(VI). Initial data made of four phase transitions ( $w$ component).

(the average of $w_{0}$ is 0 , as is the average of $v_{0}$ ), we now observe a double $N$-wave pattern on the linearized Riemann invariants, and for large times, the solution does not seem to converge to a solution made of phase transitions with equal propagation speeds. Asymptotically, the solution does contain two phase transitions, that is, exactly the same number as there were in the initial data.

We give another test of interest which demonstrates that the number of phase transitions may be asymptotically larger than the one in the initial data. Consider the periodic initial data

$$
\left(v_{0}(x), w_{0}(x)\right)= \begin{cases}(-0.3,0.4), & x<-0.3, \\ (0.15,-0.2), & -0.3<x<-0.3+2 / 3, \\ (-0.3,0.4), & x>-0.3+2 / 3 .\end{cases}
$$

We plotted the $w$ component of the solution in Figure $7 \mathrm{C}(\mathrm{V})$. For large times, we observe a solution made of four phase transitions with coinciding propagation speeds.

To conclude this section, consider a periodic initial data made of four phase transitions, precisely

$$
\left(v_{0}(x), w_{0}(x)\right)= \begin{cases}(0.1,0.4), & x<-0.4, \\ (0.2,-0.3), & -0.4<x<-0.1, \\ (0,0.4), & -0.1<x<0.1, \\ (0,-0.2), & 0.1<x<0.3, \\ (0.1,0.4), & x>0.3 .\end{cases}
$$

We plotted the $w$ component of the numerical solution in Figure 7C(VI). Observe that here none of the averages of $v_{0}$ or $w_{0}$ is zero. For sufficiently large times, we obtain a solution made of two phase transitions (propagating with the same speed), so that the number of transitions has decreased with respect to the one in the initial data.

Test 8: Mesh refinements

Consider the initial data

$$
\left(v_{0}(x), w_{0}(x)\right)= \begin{cases}(1,3), & x<0 \\ (0.2,-4), & x>0\end{cases}
$$



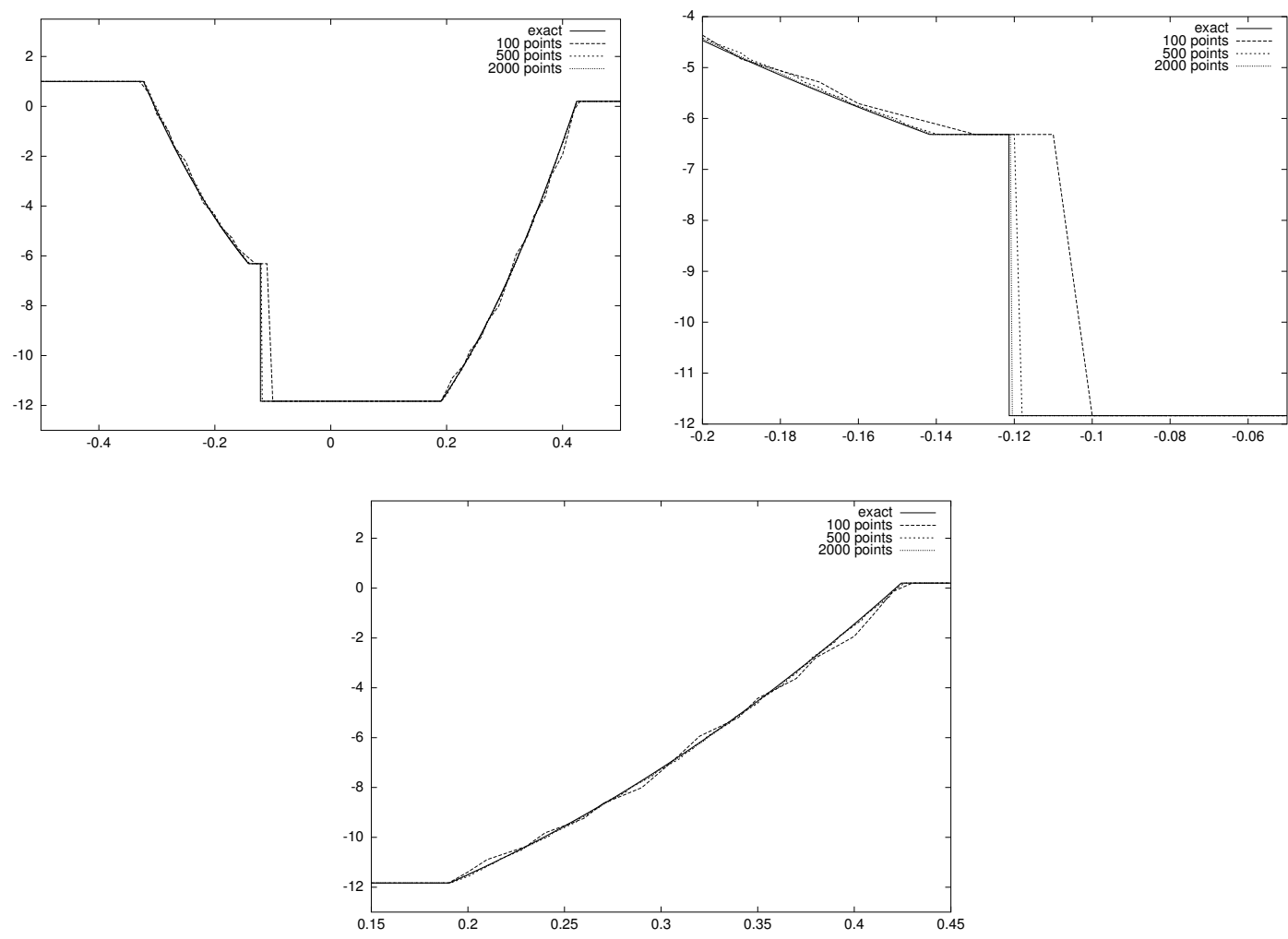

FIG. 8A. System case: convergence (global $v$ component and zooms).

We plot the numerical solution in Figures $8 \mathrm{~A}$ and $8 \mathrm{~B}$ for several mesh lengths, at time $t=0.06$ and for $\beta=2 / 3$. This test illustrates that the random choice method converges very sharply to the exact nonclassical solution of the problem under consideration.

\section{Conclusions}

In this paper, we have studied from a numerical point of view two hyperbolic models giving rise to nonclassical undercompressive shock waves, namely the scalar conservation laws and the model of elastodynamics when the flux is a cubic function. We have investigated the stability and timeasymptotic properties of classical and nonclassical entropy solutions.

(1) We have demonstrated that Glimm's scheme converges to exact solutions, even when the classical Riemann solver is replaced with a nonclassical Riemann solver.

(2) It is well known that for classical entropy solutions the total variation is a nonincreasing functional for scalar conservation laws, and for systems, it may increase only by the quadratic product of the incoming wave strengths at most [9]. This is no longer true for nonclassical solutions [21]. We have numerically observed that, under small perturbations, classical shock waves may be split into a nonclassical shock and a classical one, which is the reason why 

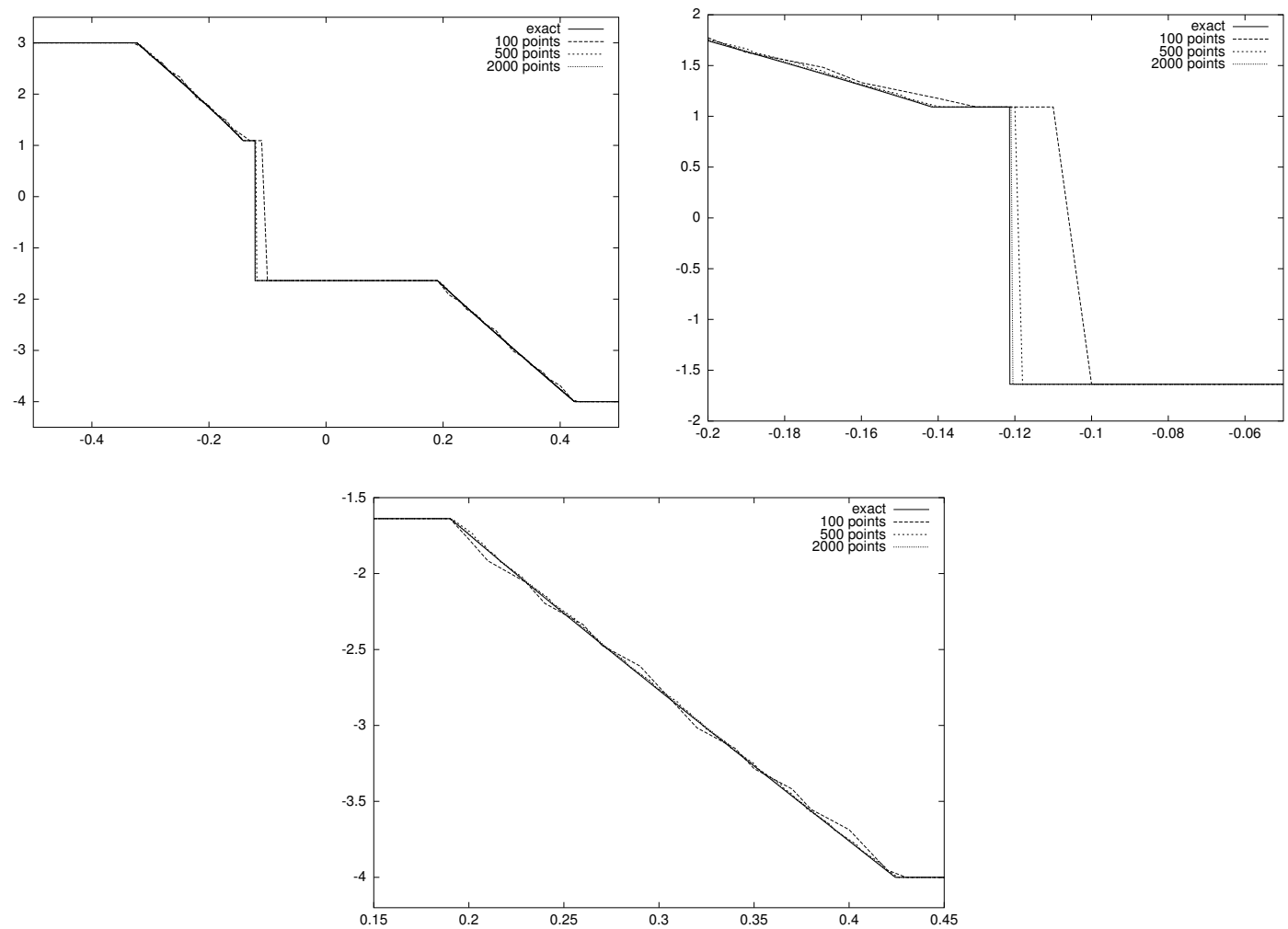

FIG. 8B. System case: convergence (global $w$-component and zooms).

the total variation of nonclassical solutions may increase drastically as the solution evolves in time. From a theoretical standpoint, this was already known when considering the nonclassical Riemann solvers (see [21] for more details). The present paper contributes to stress this feature from the numerical perspective. Glimm's scheme allows one to compute fine structures of exact solutions with high accuracy. On the other hand, finite difference schemes smooth out discontinuities, which may be disastrous when computing small-scale sensitive waves such as nonclassical shocks (and phase boundaries).

(3) We have studied the time-asymptotic behavior of periodic solutions. We found that any periodic solution asymptotically converges to a constant state and, more precisely, approaches a well known $N$-wave pattern when the average of the initial data is nonzero but approaches a "double $N$-wave pattern" if this average equals zero. In the latter, nonclassical double $\mathrm{N}$-waves contain two interfaces propagating with a speed fulfilling the Rankine-Hugoniot relation and the kinetic relation. These conclusions hold for the conservative variable in the scalar case (Section 2) and for the linearized Riemann invariants for the system (Section 3). It would be very interesting to determine this pattern analytically. It is conceivable that the existence of a double $\mathrm{N}$-wave pattern is typical of classical and nonclassical solutions of general hyperbolic systems which fail to have globally genuinely nonlinear characteristic fields. 
(4) Special attention was devoted to the maximally dissipative kinetic function, which allows us to exhibit still another interesting behavior of nonclassical entropy solutions. Generally speaking, the numerical solution converges for large times to an oscillating pattern involving only two constant values and a finite number of transitions propagating with the same speed. In addition, this number may decrease as well as increase in time.

A follow-up paper will investigate to which extent our conclusions remain valid for the hyperbolic-elliptic version of 3.1. Additional difficulties arise in this context, for instance the lack of continuous dependence and uniqueness of the nonclassical solver (see [25]), which we will have to address from the numerical standpoint.

To close this paper, we emphasize that Glimm's scheme compares favorably with numerical methods for computing nonclassical shock waves. In particular, by taking into account diffusive and dispersive terms directly within a finite difference scheme, it is possible to compute nonclassical shock waves with arbitrary accuracy and to approach a prescribed kinetic relation generated by a zero diffusion-dispersion limit. See [13], [23], [3], [4], [22]. However the accuracy deteriorates with shocks with large strength and with large-time computations.

In contrast, Glimm's scheme

- converges to the correct solution satisfying the prescribed kinetic relation for all nonclassical shock waves, even with arbitrary strength,

- is very flexible and encompasses arbitrary kinetic functions, which need not be generated by a specific diffusive-dispersive mechanism, but may be determined by experiments (for instance),

- and allows one to determine reliable large-time asymptotics of nonclassical solutions.

\section{REFERENCES}

1. Abeyaratne, R. \& Knowles, J. K. Kinetic relations and the propagation of phase boundaries in solids. Arch. Rational Mech. Anal. 114 (1991), 119-154. Zbl 0745.73001 MR 92a:73006

2. Abeyaratne, R. \& Knowles, J. K. Implications of viscosity and strain gradient effects for the kinetics of propagating phase boundaries. SIAM J. Appl. Math. 51 (1991), 1205-1221. Zbl 0764.73013 MR 92m:73011

3. Chalons, C. \& LeFloch, P. G. High-order entropy conservative schemes and kinetic relations for van der Waals fluids. J. Comput. Phys. 167 (2001), 1-23. Zbl pre01613441 MR 2002a:76113

4. Chalons, C. \& LeFloch, P. G. A fully discrete scheme for diffusive-dispersive conservation laws. Numer. Math. 89 (2001), 493-509. Zbl pre01686206 MR 2002g:65095

5. Colella, P. Glimm's method for gas dynamics. SIAM J. Sci. Stat. Comput. 3 (1982), 76-110. Zbl 0502.76073 MR 83f:76069

6. FAN, H. T. A limiting "viscosity" approach to the Riemann problem for materials exhibiting a change of phase II. Arch. Rational Mech. Anal. 116 (1992), 317-337. MR 93a:35102

7. FAN, H. T. One-phase Riemann problem and wave interactions in systems of conservation laws of mixed type. SIAM J. Math. Anal. 24 (1993), 840-865. Zbl 0804.35074 MR 94f:35082

8. FAn, H. T. \& Slemrod, M. The Riemann problem for systems of conservation laws of mixed type. Shock Induced Transitions and Phase Structures in General Media, R. Fosdick, E. Dunn, and H. Slemrod (eds.), IMA Vol. Math. Appl. 52, Springer, 1993, 61-91. Zbl 0807.76031 MR 94h:35152

9. Glimm, J. Solutions in the large for nonlinear hyperbolic systems of equations. Comm. Pure Appl. Math. 18 (1965), 697-715. Zbl 0141.28902 MR 33 \#2976

10. HATtORI, H. The Riemann problem for a van der Waals fluid with the entropy rate admissibility criterion. Arch. Rational Mech. Anal. 92 (1986), 247-263. Zbl 0673.76084 MR 87h:76085 
11. Hayes, B. T. \& LeFloch, P. G. Nonclassical shock waves and kinetic relations (II). Preprint \# 357, CMAP, Ecole Polytechnique (France), November 1996.

12. Hayes, B. T. \& LeFloch, P. G. Nonclassical shocks and kinetic relations: Scalar conservation laws. Arch. Rational Mech. Anal. 139 (1997), 1-56. Zbl 0902.76053

13. Hayes, B. T. \& LeFloch, P. G. Nonclassical shocks and kinetic relations: Finite difference schemes. SIAM J. Numer. Anal. 35 (1998), 2169-2194. Zbl 0938.35096 MR 99k:65073

14. Hayes, B. T. \& LeFloch, P. G. Nonclassical shock waves and kinetic relations: Strictly hyperbolic systems. SIAM J. Math. Anal. 31 (2000), 941-991. Zbl 0953.35095 MR 2001j:35194

15. Hou, T. Y., Rosakis, P., \& LeFloch, P. G. A level set approach to the computation of twinning and phase transition dynamics. J. Comput. Phys. 150 (1999), 302-331. Zbl 0936.74052 MR 2000a:74116

16. Jacobs, D., McKinney, W. R., \& Shearer, M. Travelling wave solutions of the modified Korteweg-deVries Burgers equation. J. Differential Equations 116 (1995), 448-467. Zbl 0820.35118 MR 96a:35170

17. JAMES, R. D. The propagation of phase boundaries in elastic bars. Arch. Rational Mech. Anal. 73 (1980), 125-158. Zbl 0443.73010 MR 80k:73009

18. LeFloch, P. G. Propagating phase boundaries: formulation of the problem and existence via the Glimm method. Arch. Rational Mech. Anal. 123 (1993), 153-197. MR 94m:35187

19. LeFloch, P. G. An introduction to nonclassical shocks of systems of conservation laws. Proc. Internat. School on Theory and Numerics for Conservation Laws (Freiburg-Littenweiler, 1997), D. Kröner, M. Ohlberger and C. Rohde (eds.), Lecture Notes in Comput. Sci. Engrg., Springer, 1998, 28-72. Zbl 0929.35088 MR 2001b:35209

20. LeFloch, P. G. Computational methods for propagating phase boundaries. Intergranular and Interphase Boundaries in Materials: iib95 (Lisbon, 1995), A. C. Ferro, J. P. Conde and M. A. Fortes (eds.), Materials Science Forum Vols. 207-209, 1996, 509-515.

21. LeFloch, P. G. Hyperbolic Systems of Conservation Laws: The Theory of Classical and Nonclassical Shock Waves, ETH Lecture Notes Series, Birkhäuser, 2002. MR 1927887

22. LeFloch, P. G., Mercier, J.-M., \& Rohde, C. Fully discrete entropy conservative schemes of arbitrary order. SIAM J. Numer. Anal., to appear (2003).

23. LeFloch, P. G. \& Rohde, C. High-order schemes, entropy inequalities, and nonclassical shocks. SIAM J. Numer. Anal. 37 (2000), 2023-2060. Zbl 0959.35117 MR 2001e:65139

24. LeFloch, P. G. \& Thanh, M. D. Nonclassical Riemann solvers and kinetic relations I: An hyperbolic model of elastodynamics. Z. Angew. Math. Phys. 52 (2001), 597-619. Zbl pre01656068 MR 2002g:35143

25. LeFloch, P. G. \& Thanh, M. D. Nonclassical Riemann solvers and kinetic relations. II. An hyperbolic-elliptic model of phase transitions. Proc. Roy. Soc. Edinburgh 131 (2001), 1-39.

26. LiU, T.-P. The deterministic version of the Glimm scheme. Comm. Math. Phys. 57 (1977), 135-148. Zbl 0376.35042 MR 57 \#10259

27. SheARer, M. The Riemann problem for a class of conservation laws of mixed type. J. Differential Equations 46 (1982), 426-443. Zbl 0465.35063 MR 84a:35164

28. SheARER, M. \& YAng, Y. The Riemann problem for a system of conservation laws of mixed type with a cubic nonlinearity. Proc. Roy. Soc. Edinburgh Sect. A 125 (1995), 675-699. Zbl 0843.35064 MR 96h:35123

29. Slemrod, M. Admissibility criteria for propagating phase boundaries in a van der Waals fluid. Arch. Rational Mech. Anal. 81 (1983), 301-315. Zbl 0505.76082 MR 84a:76030

30. Slemrod, M. The viscosity-capillarity criterion for shocks and phase transitions. Arch. Rational Mech. Anal. 83 (1983), 333-361. Zbl 0531.76069 MR 85i:76033

31. Slemrod, M. Dynamic phase transitions in a van der Waals fluid. J. Differential Equations 52 (1984), 1-23. Zbl 0487.76006 MR 85e:76040 
32. Slemrod, M. A limiting "viscosity" approach to the Riemann problem for materials exhibiting change of phase. Arch. Rational Mech. Anal. 105 (1989), 327-365. Zbl 0701.35101 MR 89m:35186

33. TRUSKinOvsky, L. Kinks versus shocks. Shock Induced Transitions and Phase Structures in General Media, R. Fosdick, E. Dunn, and M. Slemrod (eds.), IMA Vol. Math. Appl. 52, Springer, New York, 1993, 185-229. Zbl 0818.76036 MR 94j:35103

34. TRuskinovsky, L. About the "normal growth" approximation in the dynamical theory of phase transitions. Contin. Mech. Thermodyn. 6 (1994), 185-208. Zbl 0877.73006 MR 95c:80006

35. Zhong, X.-G., Hou, T. Y., \& LeFloch, P. G. Computational methods for propagating phase boundaries. J. Comput. Phys. 124 (1996), 192-216. Zbl 0855.73080 MR 96k:73093 